\title{
Finite blocklength converse bounds for quantum channels
}

\author{
William Matthews \\ Statistical Laboratory, University of Cambridge, Wilberforce Road, Cambridge, CB3 0WB Englan屯* \\ Stephanie Wehner \\ Centre for Quantum Technologies, National University of Singapore, 3 Science Drive 2, Singapore, 117543
}

\begin{abstract}
We derive upper bounds on the rate of transmission of classical information over quantum channels by block codes with a given blocklength and error probability, for both entanglement-assisted and unassisted codes, in terms of a unifying framework of quantum hypothesis testing with restricted measurements. Our bounds do not depend on any special property of the channel (such as memorylessness) and generalise both a classical converse of Polyanskiy, Poor, and Verdú as well as a quantum converse of Renner and Wang, and have a number of desirable properties. In particular our bound on entanglement-assisted codes is a semidefinite program and for memoryless channels its large blocklength limit is the well known formula for entanglement-assisted capacity due to Bennett, Shor, Smolin and Thapliyal.
\end{abstract}

\section{INTRODUCTION}

This work is concerned with the transmission of classical information over quantum channels by means of block codes. This is a central subject of study in quantum information theory, and the asymptotic rates of transmission in the large blocklength limit, for various types of code and channel, are the subject of celebrated theorems and intriguing open problems. A more fundamental problem, of both theoretical and practical interest, is to obtain upper (or converse) and lower (or achievability) bounds on the optimal transmission rate for a given error probability $\epsilon$ and finite blocklength $n$.

Without assumptions on the structure of the operation implemented by $n$ channel uses (e.g. independence), there is only a notational difference between coding for $n$ uses of a channel and coding for one use of a larger composite channel, so bounds which apply in this setting are also known as 'one-shot' bounds. These are the subject of a number of recent results in quantum information [1 [5] and remain an active topic of research in classical information [6, 7]. All bounds referred to in the remainder of this introduction are of this type.

Mosonyi and Datta [1, Wang and Renner [2] and Renes and Renner 3 , have given converse and achievability bounds for classical-quantum channels. In [4 Datta and Hsieh derive converse and achievability results for entanglement-assisted coding over quantum channels in terms of smoothed min- and max-entropies.

Polyanskiy, Poor and Verdú [6] identified a very general approach to classical converse bounds which they call the 'meta-converse'. The bounds they obtain are given in terms of a classical hypothesis testing problem. In this paper we further generalise this approach to the quantum setting, obtaining novel converse bounds for both

\footnotetext{
*Electronic address: will@northala.net, This paper was presented
} in part at Quantum Information Processing 2013 entanglement-assisted and unassisted coding over general quantum channels. Our bounds are given in terms of a quantum hypothesis testing problem on a bipartite system. In the bounds for unassisted codes the measurements used for the hypothesis test obey certain locality restrictions with respect to the bipartition.

Section II gives a brief review of the mathematical framework that we work with, introduces the classes of restricted operations we use (II A) and quantum hypothesis testing with these restrictions (II B), and defines precisely entanglement-assisted and unassisted codes (II C). Section III states our converse bounds and summarises the properties of these bounds, which we establish in Section $[\mathrm{V}$ Section $\mathrm{IV}$ proves the converse bounds via a quantum 'meta-converse' result.

As an example of the application of our bound for entanglement-assisted codes, in Section VI we show how to compute it exactly for $n$ uses of a depolarising channel. In Section VII we discuss the relationship of this work to existing results on strong converse bounds for quantum channels, and to security proofs in the noisystorage model. We conclude in Section VIII, where we mention some open problems.

\section{PRELIMINARIES}

As usual, a quantum system $\mathrm{Q}$ is associated with a Hilbert space $\mathcal{H}_{\mathrm{Q}}$. By the dimension of the system $\operatorname{dim}(\mathrm{Q})$ we mean the dimension of the associated space. This work deals only with finite-dimensional systems. The space of linear maps from $\mathcal{H}_{\mathrm{Q}}$ to $\mathcal{H}_{\mathrm{R}}$, we denote by $\mathcal{L}\left(\mathcal{H}_{\mathrm{Q}}, \mathcal{H}_{\mathrm{R}}\right)$, with the abbreviation $\mathcal{L}\left(\mathcal{H}_{\mathrm{Q}}\right)$ for the space $\mathcal{L}\left(\mathcal{H}_{\mathrm{Q}}, \mathcal{H}_{\mathrm{Q}}\right)$ of linear operators on $\mathcal{H}_{\mathrm{Q}}$.

By a state of $\mathrm{Q}$ we mean a density (i.e. positive semidefinite, trace one) operator on $\mathcal{L}\left(\mathcal{H}_{\mathrm{Q}}\right)$. We denote the set of all states of $\mathrm{Q}$ by $\operatorname{states}(\mathrm{Q})$. We omit tensor products, when the subscripts make it clear on which systems the operators act e.g. $\rho_{\mathrm{A}} \sigma_{\mathrm{B}}=\rho_{\mathrm{A}} \otimes \sigma_{\mathrm{B}}$.

By a sub-operation with input system $\mathrm{A}$ and output 
system B we mean a linear map from $\mathcal{L}\left(\mathcal{H}_{\mathrm{A}}\right)$ to $\mathcal{L}\left(\mathcal{H}_{\mathrm{B}}\right)$ which is completely positive and trace non-increasing. We denote the set of these by $\operatorname{subops}(\mathrm{A} \rightarrow \mathrm{B})$. If a sub-operation is trace preserving, then it is called an operation. We denote the subset of operations in $\operatorname{subops}(\mathrm{A} \rightarrow \mathrm{B})$ by ops $(\mathrm{A} \rightarrow \mathrm{B})$.

As a completely positive map, any sub-operation $\mathcal{N}_{\mathrm{B} \mid \mathrm{A}} \in \operatorname{subops}(\mathrm{A} \rightarrow \mathrm{B})$ has a (non-unique) representation

$$
\mathcal{N}_{\mathrm{B} \mid \mathrm{A}}: X_{\mathrm{A}} \mapsto \sum_{i} M_{i} X_{\mathrm{A}} M_{i}^{\dagger}
$$

in terms of linear operators $M_{i} \in \mathcal{L}\left(\mathcal{H}_{\mathrm{A}}, \mathcal{H}_{\mathrm{B}}\right)$ called Kraus operators. The trace non-increasing condition is equivalent to $\sum_{i} M_{i}^{\dagger} M_{i} \leq \mathbb{1}_{\mathrm{A}}$, and $\mathcal{N}_{\mathrm{B} \mid \mathrm{A}}$ is trace preserving iff $\sum_{i} M_{i}^{\dagger} M_{i}=\mathbb{1}_{\mathrm{A}}$.

Given a linear map $\mathcal{N}_{\mathrm{B} \mid \mathrm{A}}: \mathcal{L}\left(\mathcal{H}_{\mathrm{A}}\right) \rightarrow \mathcal{L}\left(\mathcal{H}_{\mathrm{B}}\right)$ its adjoint map is defined to be the unique linear map $\mathcal{N}_{\mathrm{B} \mid \mathrm{A}}^{\dagger}: \mathcal{L}\left(\mathcal{H}_{\mathrm{B}}\right) \rightarrow \mathcal{L}\left(\mathcal{H}_{\mathrm{A}}\right)$ such that, for all $X_{\mathrm{A}} \in$ $\mathcal{L}\left(\mathcal{H}_{\mathrm{A}}\right)$ and $Y_{\mathrm{B}} \in \mathcal{L}\left(\mathcal{H}_{\mathrm{B}}\right)$, we have $\operatorname{Tr}_{\mathrm{B}} Y_{\mathrm{B}}^{\dagger} \mathcal{N}_{\mathrm{B} \mid \mathrm{A}} X_{\mathrm{A}}=$ $\operatorname{Tr}_{\mathrm{A}}\left(\mathcal{N}_{\mathrm{A} \mid \mathrm{B}}^{\dagger} Y_{\mathrm{B}}\right)^{\dagger} X_{\mathrm{A}}$. If $\mathcal{N}_{\mathrm{B} \mid \mathrm{A}}$ is an operation, then $\mathcal{N}_{\mathrm{A} \mid \mathrm{B}}^{\dagger}$ is completely positive and unital (i.e. it maps the identity operator to the identity operator).

Processes which produce a classical outcome in some set $W$, can be represented by an instrument. For our purposes, it suffices to consider finite $W$ and we can represent an instrument by a collection of suboperations indexed by $W,\left\{\mathcal{N}_{\mathrm{B} \mid \mathrm{A}}^{(w)}\right\}_{w \in W}$, which sum to an operation: $\sum_{w \in W} \mathcal{N}_{\mathrm{B} \mid \mathrm{A}}^{(w)} \in \operatorname{ops}(\mathrm{A} \rightarrow \mathrm{B})$. If the instrument is applied to a state $\rho_{\mathrm{A}}$ then the probability of outcome $w$ is $\operatorname{Tr}_{\mathrm{B}} \mathcal{N}_{\mathrm{B} \mid \mathrm{A}}^{(w)} \rho_{\mathrm{A}}$, and state of $\mathrm{B}$ conditioned on outcome $w$ is $\left(\mathcal{N}_{\mathrm{B} \mid \mathrm{A}}^{(w)} \rho_{\mathrm{A}}\right) /\left(\operatorname{Tr}_{\mathrm{B}} \mathcal{N}_{\mathrm{B} \mid \mathrm{A}}^{(w)} \rho_{\mathrm{A}}\right)$. If $M_{i}^{(w)}$ are Kraus operators for $\mathcal{N}_{\mathrm{B} \mid \mathrm{A}}^{(w)}$, then $\operatorname{Tr}_{\mathrm{B}} \mathcal{N}_{\mathrm{B} \mid \mathrm{A}}^{(w)} \rho_{\mathrm{A}}=\sum_{i} \operatorname{Tr}_{\mathrm{B}} M_{i}^{(w)} \rho_{\mathrm{A}}\left(M_{i}^{(w)}\right)^{\dagger}=$ $\operatorname{Tr}_{\mathrm{A}} E(w)_{\mathrm{A}} \rho_{\mathrm{A}}$ where $E(w)_{\mathrm{A}}:=\sum_{i}\left(M_{i}^{(w)}\right)^{\dagger} M_{i}^{(w)}$, and the equation follows from the linearity and cyclic property of trace. The POVM (positive operator-valued measure) $\left\{E(w)_{\mathrm{A}}\right\}_{w \in W}$ and the state $\rho_{\mathrm{A}}$ determine the distribution of the outcome. The fact that $\left\{\mathcal{N}_{\mathrm{B} \mid \mathrm{A}}^{(w)}\right\}_{w \in W}$ is an instrument implies that POVM elements satisfy $E(w)_{\mathrm{A}} \geq 0$ and $\sum_{w \in W} E(w)_{\mathrm{A}}=\mathbb{1}_{\mathrm{A}}$.

It is convenient to assume that every quantum system Q comes equipped with a canonical orthonormal basis which we call the classical basis, and whose members we denote by $|i\rangle_{\mathrm{Q}}$ for $i=1, \ldots, \operatorname{dim}(\mathrm{Q})$. Given two systems $\mathrm{Q}$ and $\tilde{\mathrm{Q}}$ of the same dimension, we denote by $\mathrm{id}_{\tilde{\mathrm{Q}} \mid \mathrm{Q}}$ the "identity" operation from Q to $\tilde{Q}$ which is defined via its action on the classical bases of the systems, thus

$$
\operatorname{id}_{\tilde{Q} \mid \mathrm{Q}}:|i\rangle\left\langle\left. j\right|_{\mathrm{Q}} \mapsto \mid i\right\rangle\left\langle\left. j\right|_{\tilde{\mathrm{Q}}}\right.
$$

We also define the transpose map on a system $\mathrm{Q}$ by its action on the classical basis

$$
\mathrm{t}_{\mathrm{Q} \mid \mathrm{Q}}:|i\rangle\left\langle\left. j\right|_{\mathrm{Q}} \mapsto \mid j\right\rangle\left\langle\left. i\right|_{\mathrm{Q}}\right.
$$

It is important to note that $\mathrm{t}_{\mathrm{Q} \mid \mathrm{Q}}$ is positive but not completely positive, so it is not an operation. Given an operator $X_{\mathrm{Q}}$ we will also make use of the standard notation for its transpose $X_{\mathrm{Q}}^{\mathrm{T}}:=\mathrm{t}_{\mathrm{Q} \mid \mathrm{Q}} X_{\mathrm{Q}}$. Similarly, the complex conjugate $X_{\mathrm{Q}}^{*}$ of $X_{\mathrm{Q}}$ is defined by taking the classical basis to be real. Therefore, the adjoint $X_{\mathrm{Q}}^{\dagger}$ of an operator $X_{\mathrm{Q}}$ satisfies $X_{\mathrm{Q}}^{\dagger}=\left(X_{\mathrm{Q}}^{\mathrm{T}}\right)^{*}$.

\section{A. Classes of operation on bipartite systems}

Let $\operatorname{ops}^{\boldsymbol{\Omega}}\left(\mathrm{A}: \mathrm{B} \rightarrow \mathrm{A}^{\prime}: \mathrm{B}^{\prime}\right)$ denote the set of all operations taking states of the bipartite system $\mathrm{A}: \mathrm{B}$ to states of $\mathrm{A}^{\prime}: \mathrm{B}^{\prime}$, which belong to class $\boldsymbol{\Omega}$. We insert the colon to make explicit the relevant bipartitions of the input and output systems.

We call an operation a measurement operation if, for any input, its output is diagonal in the classical basis of its output system(s). It is worth emphasising that the classical basis of a composite system is the product basis formed from the classical bases of its constituents. We denote the subset of measuring operations in $\mathbf{o p s}^{\boldsymbol{\Omega}}(\mathrm{A}$ : $\left.\mathrm{B} \rightarrow \mathrm{A}^{\prime}: \mathrm{B}^{\prime}\right)$ by $\operatorname{mops}^{\boldsymbol{\Omega}}\left(\mathrm{A}: \mathrm{B} \rightarrow \mathrm{A}^{\prime}: \mathrm{B}^{\prime}\right)$. Measuring operations are also called "quantum-classical", or "q-c", operations in the literature.

An operation $\mathcal{N}_{\mathrm{A}^{\prime} \mathrm{B}^{\prime} \mid \mathrm{AB}} \in \operatorname{ops}\left(\mathrm{A}: \mathrm{B} \rightarrow \mathrm{A}^{\prime}: \mathrm{B}^{\prime}\right)$ belongs to the class PPT if it is positive-partial-transpose preserving, i.e. if $t_{\mathrm{B}^{\prime} \mid \mathrm{B}^{\prime}} \mathcal{N}_{\mathrm{A}^{\prime} \mathrm{B}^{\prime} \mid \mathrm{AB}} \mathrm{t}_{\mathrm{B} \mid \mathrm{B}}$ is completely positive.

$\mathcal{N}_{\mathrm{A}^{\prime} \mathrm{B}^{\prime} \mid \mathrm{AB}}$ belongs to $\mathbf{L C} \mathbf{1}$ if it can be implemented by local operations and one-way classical communication from Alice to Bob: Alice performs any instrument on her side, generating a classical outcome $a$ which she sends to Bob. Bob uses $a$ to determine which operation he applies. Such an operation can be written in the form

$$
\mathcal{N}_{\mathrm{A}^{\prime} \mathrm{B}^{\prime} \mid \mathrm{AB}}=\sum_{a} \mathcal{F}_{\mathrm{A}^{\prime} \mid \mathrm{A}}^{(a)} \mathcal{D}_{\mathrm{B}^{\prime} \mid \mathrm{B}}^{(a)}
$$

where, for each $a \mathcal{F}_{\mathrm{A}^{\prime} \mid \mathrm{A}}^{(a)} \in \operatorname{subops}\left(\mathrm{A} \rightarrow \mathrm{A}^{\prime}\right), \sum_{a} \mathcal{F}_{\mathrm{A}^{\prime} \mid \mathrm{A}}^{(a)} \in$ $\operatorname{ops}\left(\mathrm{A} \rightarrow \mathrm{A}^{\prime}\right)$, and $\mathcal{D}_{\mathrm{B}^{\prime} \mid \mathrm{B}}^{(a)} \in \operatorname{ops}\left(\mathrm{B} \rightarrow \mathrm{B}^{\prime}\right)$. Throughout, we will omit tensor products between operations when it is clear which systems they act on from the subscripts.

$\mathcal{N}_{\mathrm{A}^{\prime} \mathrm{B}^{\prime} \mid \mathrm{AB}}$ belongs to $\mathbf{L}$ if it can be implemented by local operations and shared randomness, which means it can be written

$$
\mathcal{N}_{\mathrm{A}^{\prime} \mathrm{B}^{\prime} \mid \mathrm{AB}}=\sum_{r} p_{r} \mathcal{F}_{\mathrm{A}^{\prime} \mid \mathrm{A}}^{(r)} \mathcal{D}_{\mathrm{B}^{\prime} \mid \mathrm{B}}^{(r)}
$$

where $\mathcal{F}_{\mathrm{A}^{\prime} \mid \mathrm{A}}^{(r)} \in \operatorname{ops}\left(\mathrm{A} \rightarrow \mathrm{A}^{\prime}\right)$ and $\mathcal{D}_{\mathrm{B}^{\prime} \mid \mathrm{B}}^{(r)} \in \operatorname{ops}\left(\mathrm{B} \rightarrow \mathrm{B}^{\prime}\right)$ and $p_{r}$ is the probability of the shared random variable being equal to $r$.

These classes of operations are all closed under com-

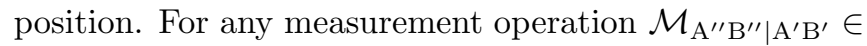
$\operatorname{mops}^{\Omega}\left(\mathrm{A}^{\prime}: \mathrm{B}^{\prime} \rightarrow \mathrm{A}^{\prime \prime}: \mathrm{B}^{\prime \prime}\right)$ and operation 
$\mathcal{W}_{\mathrm{A}^{\prime} \mathrm{B}^{\prime} \mid \mathrm{AB}} \in \operatorname{mops}^{\boldsymbol{\Omega}}\left(\mathrm{A}: \mathrm{B} \rightarrow \mathrm{A}^{\prime}: \mathrm{B}^{\prime}\right)$ (where $\boldsymbol{\Omega} \in$ $\{\mathbf{L}, \mathbf{L C 1}, \mathbf{P P T}, \mathbf{A L L}\})$ we have the closure property

$$
\mathcal{M}_{\mathrm{A}^{\prime \prime} \mathrm{B}^{\prime \prime} \mid \mathrm{A}^{\prime} \mathrm{B}^{\prime}} \mathcal{W}_{\mathrm{A}^{\prime} \mathrm{B}^{\prime} \mid \mathrm{AB}} \in \operatorname{mops}^{\boldsymbol{\Omega}}\left(\mathrm{A}: \mathrm{B} \rightarrow \mathrm{A}^{\prime \prime}: \mathrm{B}^{\prime \prime}\right) .
$$

Note, however, that following a measurement operation with an operation which is not measuring won't necessarily result in a measurement operation. All the classes mentioned are closed under convex combination. Furthermore, they form a hierarchy ALL $\supset$ PPT $\supset \mathbf{L C 1} \supset$ L [8, 9].

\section{B. Quantum Hypothesis Testing with Restricted Measurements.}

In a classical hypothesis testing problem (with simple hypotheses and finite sample space) there are two hypotheses $H_{i}, i \in\{0,1\}$, of the form is 'the random variable $R$ has distribution $P^{(i)}$. A statistical test $T$ can be specified by the giving the probabilities $T(r)=$ $\operatorname{Pr}\left(\right.$ accept $\left.H_{0} \mid T, R=r\right)$.

The 'type-I error' of $T$ is

$$
\begin{aligned}
\alpha\left(P^{(0)}, T\right) & =\operatorname{Pr}\left(\text { accept } H_{1} \mid H_{0}, T\right) \\
& =1-\sum_{r} P^{(0)}(r) T(r)
\end{aligned}
$$

while the 'type-II error' of $T$ is

$$
\begin{aligned}
\beta\left(P^{(1)}, T\right) & =\operatorname{Pr}\left(\text { accept } H_{0} \mid H_{1}, T\right) \\
& =\sum_{r} P^{(1)}(r) T(r) .
\end{aligned}
$$

\section{Definition 1.}

$$
\begin{aligned}
& \beta_{\epsilon}\left(P^{(0)} \| P^{(1)}\right):= \min \beta\left(P^{(1)}, T\right) \\
& \text { subject to } \\
& \alpha\left(P^{(0)}, T\right) \leq \epsilon, \\
& \forall r: \quad 0 \leq T(r) \leq 1 .
\end{aligned}
$$

In a quantum hypothesis testing problem (with simple hypotheses) there are two hypotheses $H_{i}, i \in\{0,1\}$, of the form is 'the state of system $\mathrm{Q}$ is $\tau_{\mathrm{Q}}^{(i)}$, In order to distinguish between these situations it is necessary to perform a measurement on Q, the outcome of which is then subjected to a classical hypothesis test.

If the measurement operation is $\mathcal{M}_{\mathrm{C} \mid \mathrm{Q}}$ and the classical test has probability $T(r)$ of accepting when the outcome is $r$, then the overall probability of acceptance when the state of $\mathrm{Q}$ is $\tau_{\mathrm{Q}}$ is

$$
\sum_{r} T(r) \operatorname{Tr}_{\mathrm{C}}|r\rangle\left\langle\left. r\right|_{\mathrm{C}} \mathcal{M}_{\mathrm{C} \mid \mathrm{Q}} \tau_{\mathrm{Q}}=\operatorname{Tr}_{\mathrm{Q}} T_{\mathrm{Q}} \tau_{\mathrm{Q}}\right.
$$

where $T_{\mathrm{Q}}=\mathcal{M}_{\mathrm{Q} \mid \mathrm{C}}^{\dagger}\left(\sum_{r} T(r)|r\rangle\left\langle\left. r\right|_{\mathrm{C}}\right)\right.$ is the POVM element corresponding to acceptance of $H_{0}$. To see that it is a POVM element, we can use the fact that $0 \leq$ $\sum_{r} T(r)|r\rangle\left\langle\left. r\right|_{\mathrm{C}} \leq 1\right.$, and that the adjoint map $\mathcal{M}_{\mathrm{Q} \mid \mathrm{C}}^{\dagger}$ of an operation is completely positive and unital.

With no restriction on the measurement operation (i.e. for class ALL), $T_{\mathrm{Q}}$ can be any valid POVM element. Therefore, we are justified in defining

\section{Definition 2.}

$$
\begin{aligned}
& \beta_{\epsilon}^{\mathbf{A L L}}\left(\tau_{\mathrm{Q}}^{(0)} \| \tau_{\mathrm{Q}}^{(1)}\right):= \min \operatorname{Tr}_{\mathrm{Q}} \tau_{\mathrm{Q}}^{(1)} T_{\mathrm{Q}} \\
& \text { subject to } \\
& 1-\operatorname{Tr}_{\mathrm{Q}} \tau_{\mathrm{Q}}^{(0)} T_{\mathrm{Q}} \leq \epsilon, \\
& \forall r: \quad 0 \leq T_{\mathrm{Q}} \leq 1 .
\end{aligned}
$$

Suppose $\tau_{\mathrm{Q}}^{(0)}$ and $\tau_{\mathrm{Q}}^{(1)}$ commute, and $\left|\eta_{i}\right\rangle\left\langle\left.\eta_{i}\right|_{\mathrm{Q}}\right.$ is a common eigenbasis for them. Defining the operation

$$
\mathcal{K}_{\mathrm{Q} \mid \mathrm{Q}}: X_{\mathrm{Q}} \mapsto \sum_{i=1}^{\operatorname{dim}(\mathrm{Q})}\left|\eta_{i}\right\rangle\left\langle\left.\eta_{i}\right|_{\mathrm{Q}} X_{\mathrm{Q}} \mid \eta_{i}\right\rangle\left\langle\left.\eta_{i}\right|_{\mathrm{Q}}\right.
$$

which completely dephases the system in this eigenbasis, we have $\mathcal{K}_{\mathrm{Q} \mid \mathrm{Q}} \tau_{\mathrm{Q}}^{(0)}=\tau_{\mathrm{Q}}^{(0)}$ and $\mathcal{K}_{\mathrm{Q} \mid \mathrm{Q}} \tau_{\mathrm{Q}}^{(1)}=\tau_{\mathrm{Q}}^{(1)}$. Since $\mathcal{K}_{\mathrm{Q} \mid \mathrm{Q}}$ is its own adjoint map, this means that there a test $T_{\mathrm{Q}}$, optimal for the hypothesis test, which itself satisfies $\mathcal{K}_{\mathrm{Q} \mid \mathrm{Q}} T_{\mathrm{Q}}=T_{\mathrm{Q}}$. That is, it too is diagonal in the common eigenbasis of the two states. In particular, if both $\tau_{\mathrm{Q}}^{(0)}$ and $\tau_{\mathrm{Q}}^{(1)}$ are diagonal in the classical basis, then we can assume without loss of generality that the test is too, whereupon everything reduces to the classical case.

We extend this definition to restricted measurements on bipartite systems by

Definition 3. For $\Omega \neq$ ALL,

$$
\beta_{\epsilon}^{\boldsymbol{\Omega}}\left(\tau_{\mathrm{AB}}^{(0)} \| \tau_{\mathrm{AB}}^{(1)}\right):=\inf \beta_{\epsilon}\left(\mathcal{M}_{\mathrm{A}^{\prime} \mathrm{B}^{\prime} \mid \mathrm{AB}} \tau_{\mathrm{AB}}^{(0)} \| \mathcal{M}_{\mathrm{A}^{\prime} \mathrm{B}^{\prime} \mid \mathrm{AB}} \tau_{\mathrm{AB}}^{(1)}\right)
$$

subject to

$$
\begin{aligned}
& \mathcal{M}_{\mathrm{A}^{\prime} \mathrm{B}^{\prime} \mid \mathrm{AB}} \in \operatorname{mops}^{\boldsymbol{\Omega}}\left(\mathrm{A}: \mathrm{B} \rightarrow \mathrm{A}^{\prime}: \mathrm{B}^{\prime}\right), \\
& \text { for arbitrary } \mathrm{A}^{\prime}, \mathrm{B}^{\prime} \text {. }
\end{aligned}
$$

That is, we reduce the quantum to the classical case by optimising over all measurement operations belonging to the allowed class $\Omega$. Based on this quantity, we define the $\boldsymbol{\Omega}$-hypothesis-testing relative entropy

$$
D_{\epsilon}^{\boldsymbol{\Omega}}\left(\tau_{\mathrm{AB}}^{(0)} \| \tau_{\mathrm{AB}}^{(1)}\right):=-\log \beta_{\epsilon}^{\boldsymbol{\Omega}}\left(\tau_{\mathrm{AB}}^{(0)} \| \tau_{\mathrm{AB}}^{(1)}\right)
$$

A trivial but very useful result is the following

Proposition 4 (Data processing inequality). If $\boldsymbol{\Omega}$ is closed under composition, then for any operation $\mathcal{N}_{\mathrm{A}^{\prime} \mathrm{B}^{\prime} \mid \mathrm{AB}} \in \operatorname{ops}^{\boldsymbol{\Omega}}\left(\mathrm{A}: \mathrm{B} \rightarrow \mathrm{A}^{\prime}: \mathrm{B}^{\prime}\right)$

$$
D_{\epsilon}^{\boldsymbol{\Omega}}\left(\mathcal{N}_{\mathrm{A}^{\prime} \mathrm{B}^{\prime} \mid \mathrm{AB}} \tau_{\mathrm{AB}}^{(0)} \| \mathcal{N}_{\mathrm{A}^{\prime} \mathrm{B}^{\prime} \mid \mathrm{AB}} \tau_{\mathrm{AB}}^{(1)}\right) \leq D_{\epsilon}^{\boldsymbol{\Omega}}\left(\tau_{\mathrm{AB}}^{(0)} \| \tau_{\mathrm{AB}}^{(1)}\right)
$$


Corollary 5. If there is also an operation $\mathcal{N}_{\mathrm{AB} \mid \mathrm{A}^{\prime} \mathrm{B}^{\prime}}^{\prime} \in \operatorname{ops}^{\Omega}\left(\mathrm{A} \quad: \quad \mathrm{B} \rightarrow \mathrm{A}^{\prime} \quad: \quad \mathrm{B}^{\prime}\right)$ such that $\mathcal{N}_{\mathrm{AB} \mid \mathrm{A}^{\prime} \mathrm{B}^{\prime}}^{\prime} \mathcal{N}_{\mathrm{A}^{\prime} \mathrm{B}^{\prime} \mid \mathrm{AB}} \tau_{\mathrm{AB}}^{(0)}=\tau_{\mathrm{AB}}^{(0)}$ and $\mathcal{N}_{\mathrm{AB} \mid \mathrm{A}^{\prime} \mathrm{B}^{\prime}}^{\prime} \mathcal{N}_{\mathrm{A}^{\prime} \mathrm{B}^{\prime} \mid \mathrm{AB}} \tau_{\mathrm{AB}}^{(1)}=\tau_{\mathrm{AB}}^{(1)}$ then

$$
D_{\epsilon}^{\Omega}\left(\mathcal{N}_{\mathrm{A}^{\prime} \mathrm{B}^{\prime} \mid \mathrm{AB}} \tau_{\mathrm{AB}}^{(0)} \| \mathcal{N}_{\mathrm{A}^{\prime} \mathrm{B}^{\prime} \mid \mathrm{AB}} \tau_{\mathrm{AB}}^{(1)}\right)=D_{\epsilon}^{\Omega}\left(\tau_{\mathrm{AB}}^{(0)} \| \tau_{\mathrm{AB}}^{(1)}\right) .
$$

Suppose that the optimal (classical) hypothesis test which acts on measurement result in Definition 3 has probability $T(a, b)$ of accepting $H_{0}$ when the measurement operation produces the outcome $|a\rangle\left\langle\left. a\right|_{\mathrm{A}^{\prime}} \mid b\right\rangle\left\langle\left. b\right|_{\mathrm{B}^{\prime}}\right.$. Then the overall probability of the test accepting $H_{0}$ when the state of $\mathrm{AB}$ is $\tau_{\mathrm{AB}}$ is

$$
\begin{aligned}
& \sum_{a, b} T(a, b) \operatorname{Tr}_{\mathrm{A}^{\prime} \mathrm{B}^{\prime}}|a\rangle\left\langle\left. a\right|_{\mathrm{A}^{\prime}} \mid b\right\rangle\left\langle\left. b\right|_{\mathrm{B}^{\prime}} \mathcal{M}_{\mathrm{A}^{\prime} \mathrm{B}^{\prime} \mid \mathrm{AB}} \tau_{\mathrm{AB}}\right. \\
= & \operatorname{Tr}_{\mathrm{AB}} T_{\mathrm{AB}} \tau_{\mathrm{AB}}
\end{aligned}
$$

where (with $\mathcal{M}_{\mathrm{AB} \mid \mathrm{A}^{\prime} \mathrm{B}^{\prime}}^{\dagger}$ the adjoint map for $\mathcal{M}_{\mathrm{A}^{\prime} \mathrm{B}^{\prime} \mid \mathrm{AB}}$ ),

$$
T_{\mathrm{AB}}=\mathcal{M}_{\mathrm{AB} \mid \mathrm{A}^{\prime} \mathrm{B}^{\prime}}^{\dagger}\left(\sum_{a, b} T(a, b)|a\rangle\left\langle\left. a\right|_{\mathrm{A}^{\prime}} \mid b\right\rangle\left\langle\left. b\right|_{\mathrm{B}^{\prime}}\right) .\right.
$$

$T_{\mathrm{AB}}$ is the POVM element (which we call simply a 'test') corresponding to acceptance of hypothesis $H_{0}$, in some quantum hypothesis test which can be implemented by a measurement operation in $\boldsymbol{\Omega}$ followed by a classical hypothesis test in the joint outcome. We denote the set of such tests on $\mathrm{AB}$ by by $\mathrm{T}^{\boldsymbol{\Omega}}(\mathrm{A}: \mathrm{B})$.

As shown in [10, $\mathbf{T}^{\mathbf{P P T}}(\mathrm{A}: \mathrm{B})$ consists of all POVM elements $T_{\mathrm{AB}}$ (that is, $0 \leq T_{\mathrm{AB}} \leq \mathbb{1}_{\mathrm{AB}}$ ), satisfying

$$
0 \leq \mathrm{t}_{\mathrm{B} \mid \mathrm{B}} T_{\mathrm{AB}} \leq \mathbb{1}_{\mathrm{AB}} .
$$

Working through the definitions, we see that $\mathbf{T}^{\mathbf{L}}(\mathrm{A}: \mathrm{B})$ is the convex hull of all POVM elements of the form

$$
\sum_{a, b} T(a, b) E(a)_{\mathrm{A}} D(b)_{\mathrm{B}}
$$

where $\left\{E(a)_{\mathrm{A}}\right\}$ is a POVM on $\mathrm{A},\left\{D(b)_{\mathrm{B}}\right\}$ is a POVM on $\mathrm{B}$, and $0 \leq T(a, b) \leq 1$. $\mathbf{T}^{\mathbf{L C} \mathbf{1}}(\mathrm{A}: \mathrm{B})$ is the convex hull of all POVM elements of the form

$$
\sum_{a, b} T(a, b) E(a)_{\mathrm{A}} D^{a}(b)_{\mathrm{B}}
$$

where $\left\{E(a)_{\mathrm{A}}\right\}$ is a POVM on $\mathrm{A}$, and for each $a$, $\left\{D^{a}(b)_{\mathrm{B}}\right\}$ is a POVM on $\mathrm{B}$, and $0 \leq T(a, b) \leq 1$. (We can use the results of [11 to show that, given our assumption that $\mathrm{A}$ and $\mathrm{B}$ are finite dimensional, it suffices to take local POVMs with a finite number of outcomes in these last two statements.)

\section{Proposition 6.}

$$
\begin{aligned}
\beta_{\epsilon}^{\boldsymbol{\Omega}}\left(\tau_{\mathrm{AB}}^{(0)} \| \tau_{\mathrm{AB}}^{(1)}\right)= & \inf \operatorname{Tr} \tau_{\mathrm{AB}}^{(1)} T_{\mathrm{AB}} \\
& \text { subject to } \\
& \operatorname{Tr}_{\mathrm{AB}} \tau_{\mathrm{AB}}^{(0)} T_{\mathrm{AB}} \geq 1-\epsilon, \\
& T_{\mathrm{AB}} \in \mathbf{T}^{\boldsymbol{\Omega}}(\mathrm{A}: \mathrm{B}) .
\end{aligned}
$$

Remark 7. For the classes of operations considered in this paper (ALL, PPT, LC1 and $\mathbf{L}), \mathbf{T}^{\boldsymbol{\Omega}}(\mathrm{A}: \mathrm{B})$ is a closed set, and so for these classes the infimum in Proposition 6 and Definition 3 can be replaced by a minimum.

\section{Codes}

As usual, a use (or uses) of a quantum channel with input system A and output system B is represented by an operation $\mathcal{E}_{\mathrm{B} \mid \mathrm{A}} \in \operatorname{ops}(\mathrm{A} \rightarrow \mathrm{B})$.

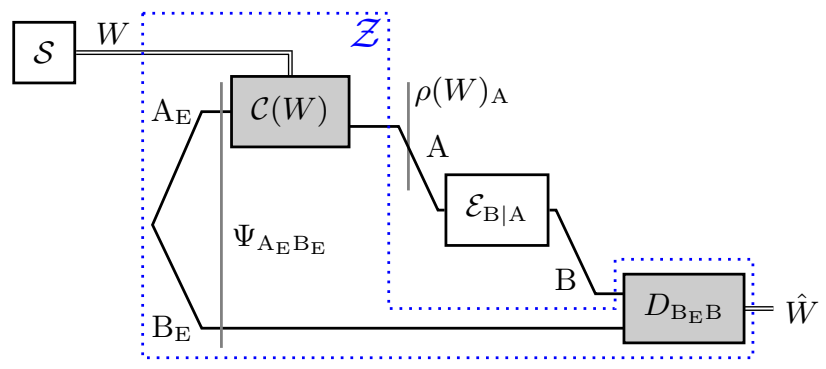

FIG. 1: An entanglement-assisted code $\mathcal{Z}$ transmitting a message $W$ produced by a source $\mathcal{S}$, via a channel use $\mathcal{E}$. The average channel input induced by the source and encoding is $\rho_{\mathrm{A}}=\sum_{w=1}^{M} \mathcal{S}(w) \rho(w)_{\mathrm{A}}$.

Definition 8. In an entanglement-assisted code of size $M$, the sender and receiver have systems $\mathrm{A}_{\mathrm{E}}$ and $\mathrm{B}_{\mathrm{E}}$ in an entangled state $\Psi_{\mathrm{A}_{\mathrm{E}} \mathrm{B}_{\mathrm{E}}}$, and for each message $w \in\{1,2, \ldots, M\}$ there is an encoding operation $\mathcal{C}(w)_{\mathrm{A} \mid \mathrm{A}_{\mathrm{E}}} \in \operatorname{ops}\left(\mathrm{A}_{\mathrm{E}} \rightarrow \mathrm{A}\right)$. Following the use(s) of the channel, the decoder performs a POVM $D_{\mathrm{B}_{\mathrm{E}} \mathrm{B}}$ on $\mathrm{B}_{\mathrm{E}} \mathrm{B}$ to obtain the decoded message. $D(\hat{w})_{\mathrm{B}_{\mathrm{E}} \mathrm{B}}$ is the POVM element corresponding the decoded message being $\hat{w}$.

Definition 9. An unassisted code can be viewed as a degenerate case of an entanglement-assisted code where the decoding measurement operates only on the channel output $\mathrm{B}$. Since $\mathrm{B}_{\mathrm{E}}$ is completely ignored, there is no loss of generality if we take $\mathrm{B}_{\mathrm{E}}$ and $\mathrm{A}_{\mathrm{E}}$ to be trivial, one-dimensional systems, so that $\mathcal{C}(w)_{\mathrm{A} \mid \mathrm{A}_{\mathrm{E}}}$ is completely specified by its output $\rho(w)_{\mathrm{A}}$ on A.

Figure 1 illustrates an entanglement-assisted code $\mathcal{Z}$ transmitting a message $W$ produced by a source $\mathcal{S}$ via a channel use $\mathcal{E}_{\mathrm{B} \mid \mathrm{A}}$. The message $W$ and the outcome $\hat{W}$ of the decoding POVM are classical random variables. The source is specified by the probabilities

$$
\mathcal{S}(w):=\operatorname{Pr}(W=w \mid \mathcal{S}) .
$$

The probability of error (which depends on the source, code and channel) is

$$
\operatorname{Pr}(\hat{W} \neq W \mid \mathcal{E}, \mathcal{Z}, \mathcal{S}) .
$$

For $M \in \mathbb{N}$ let $\mathcal{S}_{M}$ denote a source with $M$ equiprobable messages i.e. $\mathcal{S}_{M}(w)=1 / M$. 
Definition 10. We call a size $M$ code $\mathcal{Z}$ an $\left(M, \epsilon, \rho_{\mathrm{A}}\right)$ code for $\mathcal{E}$, if its average error probability for $M$ equiprobable messages satisfies $\operatorname{Pr}\left(\hat{W} \neq W \mid \mathcal{E}, \mathcal{Z}, \mathcal{S}_{M}\right) \leq \epsilon$, and the average channel input state induced by using the code with equiprobable messages is $\rho_{\mathrm{A}}$.

We denote by $M_{\epsilon}\left(\mathcal{E}_{\mathrm{B} \mid \mathrm{A}}, \rho_{\mathrm{A}}\right)$ the largest $M$ such that there is an $\left(M, \epsilon, \rho_{\mathrm{A}}\right)$ unassisted code, and by $M_{\epsilon}\left(\mathcal{E}_{\mathrm{B} \mid \mathrm{A}}\right)$ the largest $M$ such that there exists an $\rho_{\mathrm{A}}$ such that there is an $\left(M, \epsilon, \rho_{\mathrm{A}}\right)$ unassisted code.

$M_{\epsilon}^{\mathrm{E}}\left(\mathcal{E}_{\mathrm{B} \mid \mathrm{A}}, \rho_{\mathrm{A}}\right)$ and $M_{\epsilon}^{\mathrm{E}}\left(\mathcal{E}_{\mathrm{B} \mid \mathrm{A}}\right)$ denote the corresponding quantities for entanglement-assisted codes.

Remark 11. Clearly,

$$
M_{\epsilon}^{\mathrm{E}}\left(\mathcal{E}_{\mathrm{B} \mid \mathrm{A}}\right)=\max _{\rho_{\mathrm{A}} \in \operatorname{states}(\mathrm{A})} M_{\epsilon}^{\mathrm{E}}\left(\mathcal{E}_{\mathrm{B} \mid \mathrm{A}}, \rho_{\mathrm{A}}\right)
$$

and

$$
M_{\epsilon}\left(\mathcal{E}_{\mathrm{B} \mid \mathrm{A}}\right)=\max _{\rho_{\mathrm{A}} \in \operatorname{states}(\mathrm{A})} M_{\epsilon}\left(\mathcal{E}_{\mathrm{B} \mid \mathrm{A}}, \rho_{\mathrm{A}}\right)
$$

\section{SUMMARY OF RESULTS}

In 6] Polyanskiy, Poor and Verdú showed that many existing classical converse results can be easily derived from a finite blocklength converse (Theorem 27 of $[6]$ ) which we will call the PPV converse. It is obtained by a simple and conceptually appealing argument relating coding to hypothesis testing on the joint distribution of the channel input and channel output. Our bounds are given in terms of a quantum hypothesis testing problem on a bipartite system, consisting of $\mathrm{B}$ (the output system for the channel operation) and $\tilde{\mathrm{A}}$ (a copy of the input system). To compactly describe the hypotheses, we first introduce a little notation.

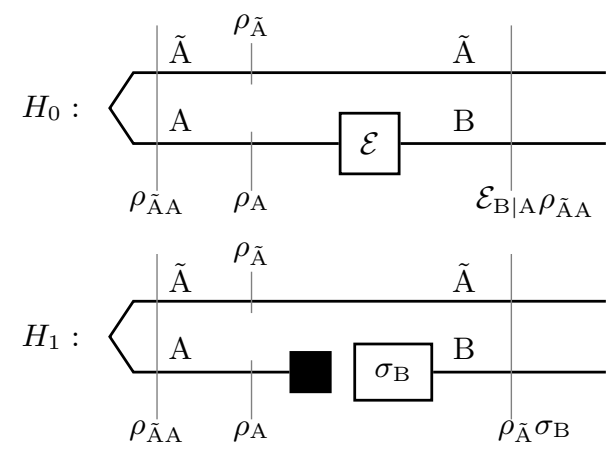

FIG. 2: The quantum hypothesis testing problem which appears in our bounds.

Definition 12. For any two systems Q, Q of the same dimension, let $\Phi_{\tilde{\mathrm{Q}} \mathrm{Q}}:=\sum_{i, j=1}^{\operatorname{dim}(\mathrm{Q})}|i\rangle_{\tilde{\mathrm{Q}}}|i\rangle_{\mathrm{Q}}\left\langle\left. j\right|_{\tilde{\mathrm{Q}}}\left\langle\left. j\right|_{\mathrm{Q}}\right.\right.$.

Remark 13. $\Phi_{\tilde{Q} Q}$ is $\operatorname{dim}(\mathrm{Q})$ times the isotropic, maximally entangled state of $\tilde{Q} Q$. Note that $\operatorname{Tr}_{\tilde{Q}} \Phi_{\tilde{Q} Q}=\mathbb{1}_{Q}$ and $\operatorname{Tr}_{\mathrm{Q}} \Phi_{\tilde{Q} \mathrm{Q}}=\mathbb{1}_{\tilde{Q}}$. We will make use, more than once, of the basic fact that for any linear operator $M_{\mathrm{Q}}$ on $\mathcal{H}_{\mathrm{Q}}$, $M_{\mathrm{Q}} \Phi_{\tilde{\mathrm{Q}} \mathrm{Q}}=M_{\tilde{\mathrm{Q}}}^{\mathrm{T}} \Phi_{\tilde{\mathrm{Q}}}$

Definition 14. Given a state $\rho_{\mathrm{A}}$, let $\tilde{\mathrm{A}}$ be a copy of system $\mathrm{A}$, and define a canonical purification of $\rho_{\mathrm{A}}$ on Aि̃ by

$$
\rho_{\tilde{\mathrm{A}} \mathrm{A}}:=\rho_{\mathrm{A}}^{\frac{1}{2}} \Phi_{\tilde{\mathrm{A}} \mathrm{A}} \rho_{\mathrm{A}}^{\frac{1}{2}} .
$$

Remark 15. By Remark 13 we have $\operatorname{Tr}_{\tilde{\mathrm{A}}} \rho_{\tilde{\mathrm{A} A}}=\rho_{\mathrm{A}}$, and we find that the marginal state of $\tilde{\mathrm{A}}$ is

$$
\rho_{\tilde{\mathrm{A}}}:=\operatorname{Tr}_{\mathrm{A}} \rho_{\tilde{\mathrm{A} A}}=\operatorname{id}_{\tilde{\mathrm{A}} \mid \mathrm{A}} \rho_{\mathrm{A}}^{\mathrm{T}} .
$$

Throughout the paper, we regard $\rho_{\tilde{\mathrm{A}} \mathrm{A}}$ and $\rho_{\tilde{\mathrm{A}}}$ as functions of $\rho_{\mathrm{A}}$.

As shown in Fig. 2 the hypotheses specify quantum states of a bipartite system $\tilde{\mathrm{AB}}$, where $\mathrm{B}$ is the output system of $\mathcal{E}_{\mathrm{B} \mid \mathrm{A}}$ and $\tilde{\mathrm{A}}$ is isomorphic to its input system. Hypothesis $H_{0}$ is that $\tilde{\mathrm{A}} \mathrm{B}$ is in the state $\mathcal{E}_{\mathrm{B} \mid \mathrm{A}} \rho_{\tilde{\mathrm{A}} \mathrm{A}}$, whereas hypothesis $H_{1}$ is that $\tilde{\mathrm{A}} \mathrm{B}$ is in the product state $\rho_{\tilde{\mathrm{A}}} \sigma_{\mathrm{B}}$.

Our main converse bounds are quantum generalisations of Theorem 27 of [6], for entanglement-assisted, and for unassisted, codes. To state them, let us introduce

\section{Definition 16.}

$$
\beta_{\epsilon}^{\boldsymbol{\Omega}}\left(\mathcal{E}_{\mathrm{B} \mid \mathrm{A}}, \rho_{\mathrm{A}}\right):=\max _{\sigma_{\mathrm{B}} \in \mathbf{s t a t e s}(\mathrm{B})} \beta_{\epsilon}^{\boldsymbol{\Omega}}\left(\mathcal{E}_{\mathrm{B} \mid \mathrm{A}} \rho_{\tilde{\mathrm{A}} \mathrm{A}} \| \rho_{\tilde{\mathrm{A}}} \sigma_{\mathrm{B}}\right),
$$

which is given in terms of Definition 3, In words, this is the minimum type-II error of all tests in class $\boldsymbol{\Omega}$ which have type-I error no greater than $\epsilon$ for the hypothesis testing problem depicted in Figure 2, maximised over all $\sigma_{\mathrm{B}}$.

The relationship between the mutual information and standard relative entropy, informs

\section{Definition 17.}

$$
\begin{aligned}
I_{\epsilon}^{\Omega}\left(\mathcal{E}_{\mathrm{B} \mid \mathrm{A}}, \rho_{\mathrm{A}}\right): & =\min _{\sigma_{\mathrm{B}} \in \operatorname{states}(\mathrm{B})} D_{\epsilon}^{\Omega}\left(\mathcal{E}_{\mathrm{B} \mid \mathrm{A}} \rho_{\tilde{\mathrm{A}} \mathrm{A}} \| \rho_{\tilde{\mathrm{A}}} \sigma_{\mathrm{B}}\right) \\
& =-\log \beta_{\epsilon}^{\Omega}\left(\mathcal{E}_{\mathrm{B} \mid \mathrm{A}}, \rho_{\mathrm{A}}\right) .
\end{aligned}
$$

We note again that $\rho_{\tilde{A} \mathrm{~A}}$ is here the canonical pure state of Definition 14 and is to be regarded as a function of $\rho_{\mathrm{A}}$.

With these and Definition 10, we can now state the main results of this paper in a compact form:

Theorem 18 (Entanglement-assisted converse).

$$
\begin{aligned}
\log M_{\epsilon}^{\mathrm{E}}\left(\mathcal{E}_{\mathrm{B} \mid \mathrm{A}}, \rho_{\mathrm{A}}\right) & \leq I_{\epsilon}^{\mathrm{ALL}}\left(\mathcal{E}_{\mathrm{B} \mid \mathrm{A}}, \rho_{\mathrm{A}}\right), \text { and so } \\
\log M_{\epsilon}^{\mathrm{E}}\left(\mathcal{E}_{\mathrm{B} \mid \mathrm{A}}\right) & \leq \max _{\rho_{\mathrm{A}} \in \operatorname{states}(\mathrm{A})} I_{\epsilon}^{\mathrm{ALL}}\left(\mathcal{E}_{\mathrm{B} \mid \mathrm{A}}, \rho_{\mathrm{A}}\right) .
\end{aligned}
$$

Theorem 19 (Unassisted converse). For any class of operations $\boldsymbol{\Omega}$ containing $\mathbf{L}$, we have

$$
\begin{aligned}
\log M_{\epsilon}\left(\mathcal{E}_{\mathrm{B} \mid \mathrm{A}}, \rho_{\mathrm{A}}\right) & \leq I_{\epsilon}^{\Omega}\left(\mathcal{E}_{\mathrm{B} \mid \mathrm{A}}, \rho_{\mathrm{A}}\right), \text { and so } \\
\log M_{\epsilon}\left(\mathcal{E}_{\mathrm{B} \mid \mathrm{A}}\right) & \leq \max _{\rho_{\mathrm{A}} \in \operatorname{states}(\mathrm{A})} I_{\epsilon}^{\Omega}\left(\mathcal{E}_{\mathrm{B} \mid \mathrm{A}}, \rho_{\mathrm{A}}\right) .
\end{aligned}
$$


The proof of both of these is given in the next section (IV). In section $\mathrm{V}$ we prove a number of properties of the bounds, which we first summarise here. The converse for entanglement-assisted codes in Theorem 18 has a number of desirable properties:

1. Like the bound of Datta and Hsieh [4, it is asymptotically tight for memoryless channels. That is to say, analysing the large block length behaviour of the bound for memoryless channels recovers the converse part of the single-letter formula for entanglement-assisted capacity proven by Bennett, Shor, Smolin and Thapliyal 12. This is shown in subsection VD

2. For a fixed blocklength, the converse of Datta and Hsieh [4] grows slowly, but without bound, as $\epsilon \rightarrow 0$ whereas our converse is a decreasing function of $\epsilon$.

3. Generalising results of Polyanskiy [7] we show that $\beta_{\epsilon}^{\mathbf{A L L}}\left(\mathcal{E}_{\mathrm{B} \mid \mathrm{A}} \rho_{\tilde{\mathrm{A}} \mathrm{A}} \| \rho_{\tilde{\mathrm{A}}} \sigma_{\mathrm{B}}\right)$ is convex in $\rho_{\mathrm{A}}$ and concave in $\sigma_{\mathrm{B}}$. This enables one to use symmetries of the channel to restrict the optimisation over $\rho_{\mathrm{A}}$ and $\sigma_{\mathrm{B}}$ to states with corresponding symmetries, as we show in subsection VE

4. In subsection $\mathrm{VA}$, we give an explicit formulation of the bound as semidefinite program (SDP) which is a natural generalisation of the linear program (LP) given in [13] for the PPV converse.

Regarding our converse for unassisted codes, Theorem 19, in subsection $\mathrm{VC}$ the Wang-Renner bound is shown to be equivalent to making the (sometimes suboptimal [7) choice $\sigma_{\mathrm{B}}=\mathcal{E}_{\mathrm{B} \mid \mathrm{A}} \rho_{\mathrm{A}}$ and taking $\boldsymbol{\Omega}$ to be the class of operations LC1 (local operations and one-way classical communication from Alice to Bob).

Since the Wang-Renner bound is asymptotically tight for the unassisted capacity (and even for the product state capacity, thus recovering the HSW theorem), the stronger bound obtained using $\mathbf{L}$ also has these properties. Unfortunately, it lacks an SDP formulation and does not possess the concavity property mentioned above. However, the formulation in terms of restricted hypothesis testing makes it clear that by moving to less restrictive conditions on the test, we might obtain weaker, but more tractable bounds. When $\boldsymbol{\Omega}$ is $\mathbf{L C 1}$, or the larger class PPT (see next section), the concavity property does hold (see Theorem 22), and we can therefore use the symmetrisation arguments.

For PPT the bound has the advantage that it can be formulated as an SDP (see subsection VA). It seems unlikely that the PPT bound is in general, asymptotically tight, but it might prove useful for certain channels.

\section{PROOF VIA METACONVERSE}

Just as in [6], our main results (Theorems 18 and 19) are consequences of a more general 'meta-converse'. Following [14, 15, we first express the general idea of the meta-converse using "generalised divergences". The hypothesis testing based bounds can be obtained by using the hypothesis-testing relative entropies as the divergences.

Let $(1-\epsilon, \epsilon)_{\mathrm{C}}$ denote a state diagonal in the classical basis with eigenvalues $(1-\epsilon, \epsilon)$ on a two-dimensional system $\mathrm{C}$ (it represents a binary probability distribution).

Suppose that, given a code $\mathcal{Z}$, we can find a measurement operation $\mathcal{T}_{\mathrm{C} \mid \mathrm{AB}}$ with a binary outcome such that, for all channel operations $\mathcal{E}_{\mathrm{B} \mid \mathrm{A}}$ from $A$ to $B$, we have

$$
\mathcal{T}_{\mathrm{C} \mid \mathrm{AB}} \mathcal{E}_{\mathrm{B} \mid \mathrm{A}} \rho_{\tilde{\mathrm{A} A}}=(1-\epsilon, \epsilon)_{\mathrm{C}},
$$

where $\epsilon$ is the error probability of the code $\mathcal{Z}$ for $\mathcal{E}$, and $\rho_{\tilde{\mathrm{AA}} \mathrm{A}}$ is a canonical purification of the average input $\rho_{\mathrm{A}}$ made to the channel by the encoder (see Definition 14).

Taking a reference channel operation $\mathcal{F}_{\mathrm{B} \mid \mathrm{A}}$ for which the error probability $f$ obtained by the code is known (or bounded) and a measure of state distinguishability (a "generalised divergence") $\mathbf{d}$ which is non-increasing under the operation $\mathcal{T}$ we have

$$
\begin{aligned}
& \mathbf{d}\left((1-\epsilon, \epsilon)_{\mathrm{C}},(1-f, f)_{\mathrm{C}}\right) \\
& \quad=\mathbf{d}\left(\mathcal{T}_{\mathrm{C} \mid \mathrm{AB}} \mathcal{E}_{\mathrm{B} \mid \mathrm{A}} \rho_{\tilde{\mathrm{A}} \mathrm{A}}, \mathcal{T}_{\mathrm{C} \mid \mathrm{AB}} \mathcal{F}_{\mathrm{B} \mid \mathrm{A}} \rho_{\tilde{\mathrm{A} A}}\right) \\
& \quad \leq \mathbf{d}\left(\mathcal{E}_{\mathrm{B} \mid \mathrm{A}} \rho_{\tilde{\mathrm{A} A}}, \mathcal{F}_{\mathrm{B} \mid \mathrm{A}} \rho_{\tilde{\mathrm{A}} \mathrm{A}}\right)
\end{aligned}
$$

bounding the difference between $\epsilon$ and the known quantity $f$.

In the classical meta-converse of Polyanskiy et al. 6] $\rho_{\mathrm{A}}=\sum_{x} p(x)|x\rangle\left\langle\left. x\right|_{\mathrm{A}}\right.$ is the classical distribution on the input alphabet induced by the encoding of equiprobable messages, and $\mathcal{E}_{\mathrm{B} \mid \mathrm{A}} \rho_{\tilde{\mathrm{A}} \mathrm{A}}$ is the joint probability distribution over the input and output alphabets induced by the use of the channel. In Wang-Renner and in Hayashi [16, $\mathcal{E}_{\mathrm{B} \mid \mathrm{A}}$ is a classical-quantum (c-q) channel which takes input symbol $x$ to some output state $\tau(x)_{\mathrm{B}}$. In these bounds $\rho_{\mathrm{A}}$ is again a probability distribution over the input symbols, while $\mathcal{E}_{\mathrm{B} \mid \mathrm{A}} \rho_{\tilde{\mathrm{A}} \mathrm{A}}$ is now the quantum state

$$
\mathcal{E}_{\mathrm{B} \mid \mathrm{A}} \rho_{\tilde{\mathrm{A}} \mathrm{A}}=\sum_{x} p(x)|x\rangle\left\langle\left. x\right|_{\tilde{\mathrm{A}}} \otimes \tau(x)_{\mathrm{B}}\right.
$$

where $\tau(x)_{\mathrm{B}}$ is the output of the classical-quantum channel on the input symbol $x$. If a code of size $M$ is used with a channel operation whose output is a fixed state $\sigma$, independent of its input, its error probability is $1 / M$. Hayashi and Wang-Renner implicitly use an such an operation for $\mathcal{F}_{\mathrm{B} \mid \mathrm{A}}$ with $\sigma_{\mathrm{B}}=\mathcal{E}_{\mathrm{B} \mid \mathrm{A}} \rho_{\mathrm{A}}$. In Theorem 27 of Polyanskiy et al. [6] the bound is optimised over all such operations. Hayashi uses the quantum relative Rényi entropy for $\mathbf{d}$ while Wang-Renner and Polyanskiy et al. use the (unrestricted) hypothesis-testing relative entropy, which itself depends on $\epsilon$.

We first show how to construct from an entanglementassisted code a measurement operation satisfying (47).

Proposition 20. From any entanglement-assisted code $\mathcal{Z}$ and source $\mathcal{S}$, such that the average input state is $\rho_{\mathrm{A}}$, 
one can construct a test $T_{\tilde{\mathrm{A}} \mathrm{B}} \in \mathbf{T}(\tilde{\mathrm{A}}: \mathrm{B})$ such that, for all $\mathcal{E}_{\mathrm{B} \mid \mathrm{A}} \in \operatorname{ops}(\mathrm{A} \rightarrow \mathrm{B})$,

$$
\operatorname{Pr}(\hat{W}=W \mid \mathcal{E}, \mathcal{Z}, \mathcal{S})=\operatorname{Tr}_{\tilde{\mathrm{A}} \mathrm{B}} T_{\tilde{\mathrm{A}} \mathrm{B}} \mathcal{E}_{\mathrm{B} \mid \mathrm{A}} \rho_{\tilde{\mathrm{A}} \mathrm{A}} .
$$

Furthermore, if $\mathcal{Z}$ is unassisted, then $T_{\tilde{\mathrm{A}} \mathrm{B}} \in \mathbf{T}^{\mathbf{L}}(\tilde{\mathrm{A}}: \mathrm{B})$.

Proof. We consider a general entanglement-assisted code as depicted in Figure 1 and using the notation established in Definition 8 . Since it is always possible to augment $\mathrm{A}_{\mathrm{E}}$ to $\mathrm{A}_{\mathrm{E}}{ }^{\prime} \mathrm{A}_{\mathrm{E}}$, let $\Psi_{\mathrm{AE}^{\prime} \mathrm{A}_{\mathrm{E}} \mathrm{B}_{\mathrm{E}}}$ be a purification of

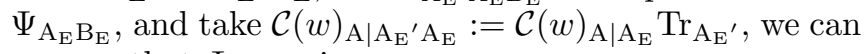
assume that $\Psi_{\mathrm{A}_{\mathrm{E}} \mathrm{B}_{\mathrm{E}}}$ is pure.

Let the isometry $U(w) \in \mathcal{L}\left(\mathcal{H}_{\mathrm{A}_{\mathrm{E}}}, \mathcal{H}_{\mathrm{G}} \otimes \mathcal{H}_{\mathrm{A}}\right)$ be the Stinespring representation of the encoding map $\mathcal{C}(w)_{\mathrm{A} \mid \mathrm{A}_{\mathrm{E}}}$, where $\mathrm{G}$ is the discarded environment system. In fact, we can just take $A_{E}=G A$ so that $U(w)_{\mathrm{GA}}$ is a unitary. So, the encoding map for message $w$ is $\mathcal{C}(w)_{\mathrm{A} \mid \mathrm{GA}}: X_{\mathrm{GA}} \mapsto \operatorname{Tr}_{\mathrm{G}} U(w)_{\mathrm{GA}} X_{\mathrm{GA}} U(w)_{\mathrm{GA}}^{\dagger}$. Finally, there is no loss of generality in demanding that $\mathrm{B}_{\mathrm{E}}=\tilde{\mathrm{G}} \tilde{\mathrm{A}} \cong \mathrm{GA}$. This reformulation of the protocol of Fig. 1 is illustrated in Fig. 3 .

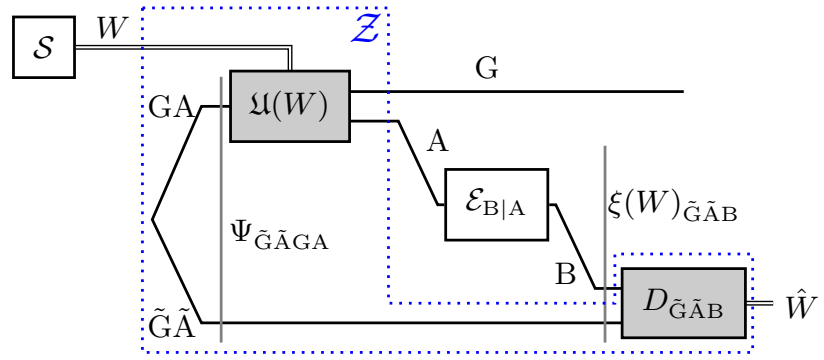

FIG. 3: Reformulation of the protocol of Fig. 1

First note that

$$
\begin{aligned}
& U(w)_{\mathrm{GA}} \Psi_{\mathrm{GA} \tilde{\mathrm{G}} \tilde{\mathrm{A}}} U(w)_{\mathrm{GA}}^{\dagger} \\
= & M(w)_{\mathrm{GA}} \Phi_{\mathrm{GA} \tilde{\mathrm{G}} \tilde{\mathrm{A}}} M(w)_{\mathrm{GA}}^{\dagger}
\end{aligned}
$$

where $M(w)_{\mathrm{GA}}:=U(w)_{\mathrm{GA}} \Psi_{\mathrm{GA}}^{\frac{1}{2}}$ and $\Psi_{\mathrm{GA}}:=$ $\operatorname{Tr}_{\tilde{\mathrm{G}} \tilde{\mathrm{A}}} \Psi_{\text {GA } \tilde{\mathrm{G}} \tilde{\mathrm{A}}}$. The state of GA after encoding if the message $W=w$ is

$$
\rho(w)_{\mathrm{GA}}=M(w)_{\mathrm{GA}} M(w)_{\mathrm{GA}}^{\dagger} .
$$

Referring to the diagram, we see that

$$
\begin{aligned}
& \operatorname{Pr}(\hat{W}=\hat{w} \mid W=w, \mathcal{E}, \mathcal{Z}) \\
= & \operatorname{Tr}_{\tilde{\mathrm{G}} \tilde{\mathrm{A}} \mathrm{B}} D(\hat{w})_{\tilde{\mathrm{G}} \tilde{\mathrm{A}} \mathrm{B}} \xi(w)_{\tilde{\mathrm{G}} \tilde{\mathrm{A}} \mathrm{B}}
\end{aligned}
$$

where $D(\hat{w})_{\tilde{\mathrm{G}} \tilde{A} \mathrm{~B}}$ is the POVM element corresponding to the decoded message being $\hat{w}$, and where

$$
\begin{aligned}
\xi(w)_{\tilde{\mathrm{G}} \tilde{\mathrm{AB}}} & =\operatorname{Tr}_{\mathrm{G}} \mathcal{E}_{\mathrm{B} \mid \mathrm{A}}\left[M(w)_{\mathrm{GA}} \Phi_{\mathrm{GA} \tilde{\mathrm{G}} \tilde{\mathrm{A}}} M(w)_{\mathrm{GA}}^{\dagger}\right] \\
& =\operatorname{Tr}_{\mathrm{G}} M(w)_{\tilde{\mathrm{G}} \tilde{\mathrm{A}}}^{\mathrm{T}} \Phi_{\tilde{\mathrm{G}} \mathrm{G}}\left(\mathcal{E}_{\mathrm{B} \mid \mathrm{A}} \Phi_{\tilde{\mathrm{A}} \mathrm{A}}\right) M(w)_{\tilde{\mathrm{G}} \tilde{\mathrm{A}}}^{*} \\
& =M(w)_{\tilde{\mathrm{G}} \tilde{\mathrm{A}}}^{\mathrm{T}} \mathbb{1}_{\tilde{\mathrm{G}}}\left(\mathcal{E}_{\mathrm{B} \mid \mathrm{A}} \Phi_{\tilde{\mathrm{A}} \mathrm{A}}\right) M(w)_{\tilde{\mathrm{G}} \tilde{\mathrm{A}}}^{*} \cdot
\end{aligned}
$$

Here we have used the facts noted in Remark 13 and that $\Phi_{\mathrm{GA} \tilde{\mathrm{G}} \tilde{\mathrm{A}}}=\Phi_{\tilde{\mathrm{A} A}} \Phi_{\tilde{\mathrm{G}} \mathrm{G}}$. The probability of successful decoding is

$$
\begin{aligned}
& \operatorname{Pr}(\hat{W}=W \mid \mathcal{E}, \mathcal{Z}, \mathcal{S}) \\
= & \sum_{w=1}^{M} \mathcal{S}(w) \operatorname{Tr}_{\tilde{\mathrm{G}} \tilde{\mathrm{A}} \mathrm{B}} D(w)_{\tilde{\mathrm{G}} \tilde{\mathrm{A}} \mathrm{B}} \xi(w)_{\tilde{\mathrm{G}} \tilde{\mathrm{A}} \mathrm{B}} \\
= & \operatorname{Tr}_{\tilde{\mathrm{G}} \tilde{\mathrm{A}} \mathrm{B}} R_{\tilde{\mathrm{G}} \tilde{\mathrm{A}} \mathrm{B}} \mathcal{E}_{\mathrm{B} \mid \mathrm{A}} \Phi_{\tilde{\mathrm{A}} \mathrm{A}}
\end{aligned}
$$

where

$$
R_{\tilde{\mathrm{G}} \tilde{\mathrm{A}} \mathrm{B}}:=\sum_{w=1}^{M} \mathcal{S}(w) M(w)_{\tilde{\mathrm{G}} \tilde{\mathrm{A}}}^{*} D(w)_{\tilde{\mathrm{G}} \tilde{\mathrm{A}} \mathrm{B}} M(w)_{\tilde{\mathrm{G}} \tilde{\mathrm{A}}}^{\mathrm{T}} .
$$

Since $R_{\tilde{\mathrm{G}} \tilde{A} \mathrm{~B}}$ is given by a completely positive map (with Kraus operators $\sqrt{\mathcal{S}(w)} M(w)_{\tilde{\mathrm{G}} \tilde{\mathrm{A}}}^{*}$ ) acting on $D_{\tilde{\mathrm{G}} \tilde{\mathrm{A}} \mathrm{B}}$, which satisfies $0 \leq D_{\tilde{\mathrm{G}} \tilde{A} \mathrm{~B}} \leq \mathbb{1}_{\tilde{\mathrm{G}} \tilde{\mathrm{A}} \mathrm{B}}$, we have $0 \leq R_{\tilde{\mathrm{G}} \tilde{\mathrm{A}} \mathrm{B}} \leq$ $\rho_{\tilde{\mathrm{G}} \tilde{\mathrm{A}}} \mathbb{1}_{\mathrm{B}}$, where $\rho_{\mathrm{GA}}:=\sum_{w=1}^{M} \mathcal{S}(w) \rho(w)_{\mathrm{GA}}$ is the average state of GA after encoding, $\rho_{\tilde{\mathrm{G}} \tilde{\mathrm{AA}}}:=\rho_{\mathrm{GA}}^{1 / 2} \Phi_{\mathrm{GA} \tilde{\mathrm{G}} \tilde{\mathrm{A}}} \rho_{\mathrm{GA}}^{1 / 2}$ is its canonical purification, and $\rho_{\tilde{\mathrm{G}} \tilde{\mathrm{A}}}:=\operatorname{Tr}_{\mathrm{GA}} \rho_{\tilde{\mathrm{G}} \tilde{\mathrm{A}} \mathrm{GA}}$. Therefore,

$$
T_{\tilde{\mathrm{A}} \mathrm{B}}:=\rho_{\tilde{\mathrm{A}}}^{-\frac{1}{2}} R_{\tilde{\mathrm{A}} \mathrm{B}} \rho_{\tilde{\mathrm{A}}}^{-\frac{1}{2}}
$$

satisfies $0 \leq T_{\tilde{\mathrm{AB}}} \leq \mathbb{1}_{\tilde{\mathrm{A}} \mathrm{B}}$, and

$$
\begin{aligned}
\operatorname{Pr}(\hat{W}=W \mid \mathcal{E}, \mathcal{Z}, \mathcal{S}) & =\operatorname{Tr}_{\tilde{\mathrm{A}} \mathrm{B}} \rho_{\tilde{\mathrm{A}}}^{\frac{1}{2}}\left(\mathcal{E}_{\mathrm{B} \mid \mathrm{A}} \Phi_{\tilde{\mathrm{A}} \mathrm{A}}\right) \rho_{\tilde{\mathrm{A}}}^{\frac{1}{2}} T_{\tilde{\mathrm{A}} \mathrm{B}} \\
& =\operatorname{Tr}_{\tilde{\mathrm{A}} \mathrm{B}} T_{\tilde{\mathrm{A}} \mathrm{B}} \mathcal{E}_{\mathrm{B} \mid \mathrm{A}} \rho_{\tilde{\mathrm{A}} \mathrm{A}}
\end{aligned}
$$

as promised.

As noted in the caption for Figure 1, any unassisted quantum code corresponds to restricting Bob's decoding measurement to the output system of the channel, so that

$$
\forall w \in\{1, \ldots, M\}: D(w)_{\tilde{\mathrm{G}} \tilde{\mathrm{A}} \mathrm{B}}=\mathbb{1}_{\tilde{\mathrm{G}} \tilde{\mathrm{A}}} D(w)_{\mathrm{B}} .
$$

Substituting this into 62 , we see that

$$
T_{\tilde{\mathrm{A}} \mathrm{B}}=\sum_{w=1}^{M} E(w)_{\tilde{\mathrm{A}}} D(w)_{\mathrm{B}}
$$

where the positive operators

$$
E(w)_{\tilde{\mathrm{A}}}:=S(w)\left(\rho_{\tilde{\mathrm{A}}}\right)^{-\frac{1}{2}}\left(\operatorname{Tr}_{\tilde{\mathrm{G}}} \rho(w)_{\tilde{\mathrm{G}} \tilde{\mathrm{A}}}\right)\left(\rho_{\tilde{\mathrm{A}}}\right)^{-\frac{1}{2}}
$$

satisfy

$$
\sum_{w=1}^{M} E(w)_{\tilde{\mathrm{A}}}=\rho_{\tilde{\mathrm{A}}}^{-\frac{1}{2}} \rho_{\tilde{\mathrm{A}}} \rho_{\tilde{\mathrm{A}}}^{-\frac{1}{2}} \leq \mathbb{1}_{\tilde{\mathrm{A}}} .
$$

Letting $E(0)_{\tilde{\mathrm{A}}}:=\mathbb{1}_{\tilde{\mathrm{A}}}-\rho_{\tilde{\mathrm{A}}}^{-\frac{1}{2}} \rho_{\tilde{\mathrm{A}}} \rho_{\tilde{\mathrm{A}}}^{-\frac{1}{2}}$, the operators $E(0)_{\tilde{\mathrm{A}}}, \ldots, E(M)_{\tilde{\mathrm{A}}}$ constitute a POVM, and so, for an unassisted code, $T_{\tilde{\mathrm{A}} \mathrm{B}}$ is a local test i.e. $T_{\tilde{\mathrm{A}} \mathrm{B}} \in \mathbf{T}^{\mathbf{L}}(\tilde{\mathrm{A}}: \mathrm{B})$. The local implementation is simply this: Alice performs a measurement with POVM elements $E(w)_{\tilde{\mathrm{A}}}$ and Bob performs the decoding measurement (with POVM elements $D(w)_{\mathrm{B}}$ ). If their outcomes are equal then the test accepts, the 'accepting' POVM element being given in equation 67). 
A quantum generalisation of the 'meta-converse' Theorem 26 of [6], is now straightforward:

Proposition 21 (Meta-converse). Let $\mathcal{Z}$ be an entanglement-assisted code which, when used with $\mathcal{S}$, induces the average input state $\rho_{\mathrm{A}}$, and which has success probability

$$
\operatorname{Pr}\left(\hat{W}=W \mid \mathcal{E}^{(i)}, \mathcal{Z}, \mathcal{S}\right)=1-\epsilon_{i},
$$

when used with channel operation $\mathcal{E}_{\mathrm{B} \mid \mathrm{A}}^{(i)}$, for $i \in\{0,1\}$. Consider the hypothesis testing problem where $H_{i}$ asserts that the state of $\tilde{\mathrm{A}} \mathrm{B}$ is $\mathcal{E}_{\mathrm{B} \mid \mathrm{A}}^{(i)} \rho_{\tilde{\mathrm{AA}}}$. If we accept $H_{0}$ when the test constructed from $(\mathcal{Z}, \mathcal{S})$ as in Proposition 20 accepts, then

$$
\beta=\operatorname{Tr}_{\tilde{\mathrm{A} B}} T_{\tilde{\mathrm{A}} \mathrm{B}} \mathcal{E}_{\mathrm{B} \mid \mathrm{A}}^{(1)} \rho_{\tilde{\mathrm{A}} \mathrm{A}}=1-\epsilon_{1}
$$

and

$$
1-\alpha=\operatorname{Tr}_{\tilde{\mathrm{A} B}} T_{\tilde{\mathrm{A}} \mathrm{B}} \mathcal{E}_{\mathrm{B} \mid \mathrm{A}}^{(0)} \rho_{\tilde{\mathrm{A}} \mathrm{A}}=1-\epsilon_{0} .
$$

Therefore, by definition

$$
\beta_{\epsilon_{0}}^{\mathbf{A L L}}\left(\mathcal{E}_{\mathrm{B} \mid \mathrm{A}}^{(0)} \rho_{\tilde{\mathrm{A} A}} \| \mathcal{E}_{\mathrm{B} \mid \mathrm{A}}^{(1)} \rho_{\tilde{\mathrm{A} A}}\right) \leq 1-\epsilon_{1} .
$$

Furthermore, if constraints on the code $\mathcal{Z}$ mean that $T_{\tilde{\mathrm{A}} \mathrm{B}}$ is guaranteed to belong to some class of tests $\mathbf{T}^{\Omega}(\mathrm{A}: \mathrm{B})$, then the (potentially more stringent) bound

$$
\beta_{\epsilon_{0}}^{\Omega}\left(\mathcal{E}_{\mathrm{B} \mid \mathrm{A}}^{(0)} \rho_{\tilde{\mathrm{A}} \mathrm{A}} \| \mathcal{E}_{\mathrm{B} \mid \mathrm{A}}^{(1)} \rho_{\tilde{\mathrm{A}} \mathrm{A}}\right) \leq 1-\epsilon_{1} .
$$

also applies.

We now apply the meta-converse to obtain our main results. Suppose that $\mathcal{E}_{\mathrm{B} \mid \mathrm{A}}^{(1)}$, is a completely useless channel operation which has $\mathcal{E}_{\mathrm{B} \mid \mathrm{A}}^{(1)} \rho_{\mathrm{A}}=\sigma_{\mathrm{B}}$ for all $\rho_{\mathrm{A}}$, and assume that the $M$ messages are equiprobable i.e. $\mathcal{S}=\mathcal{S}_{M}$. Then, it is easily verified that $1-\epsilon_{1}=1 / M$ (in fact any other value would imply that communication is possible in the absence of a channel.) Setting $\mathcal{E}_{\mathrm{B} \mid \mathrm{A}}^{(0)}=\mathcal{E}_{\mathrm{B} \mid \mathrm{A}}$, the hypothesis testing problem described in the Proposition above is now exactly the one shown in Figure 2. With these choices, equation (74) tells us that any $\left(M, \epsilon, \rho_{\mathrm{A}}\right)$ code whose corresponding test belongs to $\mathbf{T}^{\boldsymbol{\Omega}}(\tilde{\mathrm{A}}: \mathrm{B})$ must satisfy

$$
\max _{\sigma_{\mathrm{B}}} \beta_{\epsilon}^{\boldsymbol{\Omega}}\left(\mathcal{E}_{\mathrm{B} \mid \mathrm{A}} \rho_{\tilde{\mathrm{A} A}} \| \rho_{\tilde{\mathrm{A}}} \sigma_{\mathrm{B}}\right) \leq 1 / M .
$$

Here, we maximise over $\sigma_{\mathrm{B}}$ to obtain the best possible bound. For entanglement-assisted codes, rearranging this and using Definition 3 gives us

$$
\log M_{\epsilon}^{\mathrm{E}}\left(\mathcal{E}_{\mathrm{B} \mid \mathrm{A}}, \rho_{\mathrm{A}}\right) \leq \min _{\sigma_{\mathrm{B}}} D_{\epsilon}^{\mathrm{ALL}}\left(\mathcal{E}_{\mathrm{B} \mid \mathrm{A}} \rho_{\tilde{\mathrm{A}} \mathrm{A}} \| \rho_{\tilde{\mathrm{A}}} \sigma_{\mathrm{B}}\right)
$$

(Theorem 18) and for unassisted codes, we can write the stronger bound

$$
\log M_{\epsilon}\left(\mathcal{E}_{\mathrm{B} \mid \mathrm{A}}, \rho_{\mathrm{A}}\right) \leq \min _{\sigma_{\mathrm{B}}} D_{\epsilon}^{\mathbf{L}}\left(\mathcal{E}_{\mathrm{B} \mid \mathrm{A}} \rho_{\tilde{\mathrm{A}} \mathrm{A}} \| \rho_{\tilde{\mathrm{A}}} \sigma_{\mathrm{B}}\right)
$$

(which is Theorem 19).

\section{PROPERTIES OF THE BOUNDS}

In this section, we generalise results of Polyanskiy [7, showing that $\beta_{\epsilon}^{\boldsymbol{\Omega}}\left(\mathcal{E}_{\mathrm{B} \mid \mathrm{A}} \rho_{\tilde{\mathrm{A}} \mathrm{A}} \| \rho_{\tilde{\mathrm{A}}} \sigma_{\mathrm{B}}\right)$ is convex in $\rho_{\mathrm{A}}$ and concave in $\sigma_{\mathrm{B}}$, provided $\boldsymbol{\Omega}$ contains $\mathbf{L C} 1$. This enables one to use symmetries of the channel to restrict the optimisation over $\rho_{\mathrm{A}}$ and $\sigma_{\mathrm{B}}$ to states with corresponding symmetries, as we show in subsection VE

Let $\beta\left(T, \rho_{\tilde{\mathrm{A}}}, \sigma_{\mathrm{B}}\right):=\operatorname{Tr}_{\tilde{\mathrm{A}} \mathrm{B}} T_{\tilde{\mathrm{A}} \mathrm{B}} \rho_{\tilde{\mathrm{A}}} \sigma_{\mathrm{B}}$. This is a bilinear function of $T$ and $\sigma_{\mathrm{B}}$. Therefore, the minimum of $\beta\left(T, \rho_{\tilde{\mathrm{A}}}, \sigma_{\mathrm{B}}\right)$ over $T$ is concave in $\sigma_{\mathrm{B}}$, and since the set states(B) and the set of tests satisfying $\alpha\left(T, \rho_{\tilde{\mathrm{A}}}\right) \leq \epsilon$ are both convex, von Neumann's minimax theorem tells us that $\max _{\sigma_{\mathrm{B}}} \beta_{\epsilon}^{\Omega}\left(\mathcal{E}_{\mathrm{B} \mid \mathrm{A}} \rho_{\tilde{\mathrm{A}} \mathrm{A}}, \rho_{\tilde{\mathrm{A}}} \sigma_{\mathrm{B}}\right)$ is also equal to

$$
\min _{T_{\tilde{\mathrm{A}} \mathrm{B}} \in \boldsymbol{\Omega}} \beta^{*}(T)
$$

subject to

$$
\operatorname{Tr}_{\tilde{\mathrm{A}} \mathrm{B}} T_{\tilde{\mathrm{A} B}} \mathcal{E}_{\mathrm{B} \mid \mathrm{A}} \rho_{\tilde{\mathrm{A}} \mathrm{A}} \geq 1-\epsilon,
$$

where

$$
\begin{aligned}
\beta^{*}\left(T, \rho_{\tilde{\mathrm{A}}}\right): & =\max _{\sigma_{\mathrm{B}}} \operatorname{Tr}_{\tilde{\mathrm{A}} \mathrm{B}} T_{\tilde{\mathrm{A}} \mathrm{B}} \rho_{\tilde{\mathrm{A}}} \sigma_{\mathrm{B}} \\
& =\left\|\operatorname{Tr}_{\mathrm{A}} T_{\tilde{\mathrm{A}} \mathrm{B}} \rho_{\tilde{\mathrm{A}}}\right\|_{\infty}
\end{aligned}
$$

As noted in [7] for the classical case, $\beta^{*}(T)$ is the (worst case) probability of type II error for $T_{\tilde{\mathrm{A} B}}$ in the compound hypothesis testing problem where $H_{0}$ is still that the state is $\mathcal{E}_{\mathrm{B} \mid \mathrm{A}} \rho_{\tilde{\mathrm{A}} \mathrm{A}}$, but now $H_{1}$ is the compound hypothesis that the state belongs to the set $\left\{\rho_{\tilde{\mathrm{A}}} \sigma_{\mathrm{B}}: \sigma_{\mathrm{B}} \in \operatorname{states}(\mathrm{B})\right\}$.

Theorem 22. For any operations $\mathcal{E}_{\mathrm{B} \mid \mathrm{A}}^{(0)}$ and $\mathcal{E}_{\mathrm{B} \mid \mathrm{A}}^{(1)}$, and class of bipartite operations $\boldsymbol{\Omega}$ which contains $\mathbf{L} \mathbf{C} \mathbf{1}$, the function

$$
\left(\epsilon, \rho_{\mathrm{A}}\right) \mapsto \beta_{\epsilon}^{\boldsymbol{\Omega}}\left(\mathcal{E}_{\mathrm{B} \mid \mathrm{A}}^{(0)} \rho_{\tilde{\mathrm{A}} \mathrm{A}} \| \mathcal{E}_{\mathrm{B} \mid \mathrm{A}}^{(1)} \rho_{\tilde{\mathrm{A}} \mathrm{A}}\right)
$$

is jointly convex in $\epsilon$ and $\rho_{\mathrm{A}}$.

Proof. Suppose that we have, for all $j \in\{1, \ldots, m\}, \epsilon_{j} \in$ $[0,1], \rho_{\mathrm{A}}^{(j)} \in \operatorname{states}(\mathrm{A})$, and $\lambda_{j} \geq 0$ such that $\sum_{j=1}^{m} \lambda_{j}=$ $1, \sum_{j=1}^{m} \lambda_{j} \rho_{\mathrm{A}}^{(j)}=\rho_{\mathrm{A}}$, and $\sum_{j=1}^{m} \epsilon_{j} \rho_{\mathrm{A}}^{(j)}=\epsilon$.

Let $T(j)_{\tilde{A} \mathrm{~B}}$ be a test in $\mathbf{T}^{\boldsymbol{\Omega}}$ that achieves $\beta_{j}:=$ $\beta_{\epsilon_{j}}^{\Omega}\left(\mathcal{E}_{\mathrm{B} \mid \mathrm{A}}^{(0)} \rho_{\tilde{\mathrm{A} A}}^{(j)} \| \mathcal{E}_{\mathrm{B} \mid \mathrm{A}}^{(1)} \rho_{\tilde{\mathrm{A} A}}^{(j)}\right)$. That is to say,

$$
\begin{aligned}
& \operatorname{Tr}_{\tilde{\mathrm{A}} \mathrm{B}}(\mathbb{1}-T(j))_{\tilde{\mathrm{A}} \mathrm{B}} \mathcal{E}_{\mathrm{B} \mid \mathrm{A}}^{(0)} \rho_{\tilde{\mathrm{A} A}}^{(j)} \leq \epsilon_{j}, \\
& \text { and } \operatorname{Tr}_{\tilde{\mathrm{A}} \mathrm{B}} T(j)_{\tilde{\mathrm{A}} \mathrm{B}} \mathcal{E}_{\mathrm{B} \mid \mathrm{A}}^{(1)} \rho_{\tilde{\mathrm{A}} \mathrm{A}}^{(j)}=\beta_{j} .
\end{aligned}
$$

The claim is that

$$
\beta_{\epsilon}^{\boldsymbol{\Omega}}\left(\mathcal{E}_{\mathrm{B} \mid \mathrm{A}}^{(0)} \rho_{\tilde{\mathrm{A}} \mathrm{A}} \| \mathcal{E}_{\mathrm{B} \mid \mathrm{A}}^{(1)} \rho_{\tilde{\mathrm{A}} \mathrm{A}}\right) \leq \sum_{j=1}^{m} \lambda_{j} \beta_{j} .
$$

We shall explicitly construct a test in $\boldsymbol{\Omega}$ which demonstrates 85 . Consider the suboperations

$$
\mathcal{M}_{\tilde{\mathrm{A}} \mid \tilde{\mathrm{A}}}^{(j)}: X_{\tilde{\mathrm{A}}} \mapsto \lambda_{j} \rho_{\tilde{\mathrm{A}}}^{(j) 1 / 2} \rho_{\tilde{\mathrm{A}}}^{-1 / 2} X_{\tilde{\mathrm{A}}} \rho_{\tilde{\mathrm{A}}}^{-1 / 2} \rho_{\tilde{\mathrm{A}}}^{(j) 1 / 2}
$$


for $j \in\{1, \ldots, m\}$ and

$$
\mathcal{M}_{\tilde{\mathrm{A}} \mid \tilde{\mathrm{A}}}^{(0)}: X_{\tilde{\mathrm{A}}} \mapsto \Pi_{\tilde{\mathrm{A}}} X_{\tilde{\mathrm{A}}} \Pi_{\tilde{\mathrm{A}}}
$$

where $\Pi_{\tilde{A}}$ is the orthogonal projector onto the kernel of $\rho_{\tilde{\mathrm{A}}}$. The key property of these suboperations is that

$$
\mathcal{M}_{\tilde{\mathrm{A}} \mid \tilde{\mathrm{A}}}^{(j)} \rho_{\tilde{\mathrm{A} A}}=\left\{\begin{array}{l}
\lambda_{j} \rho_{\tilde{\mathrm{AA}}}^{(j)} \text { for } j \in\{1, \ldots, m\}, \\
0 \text { for } j=0 .
\end{array}\right.
$$

Since

$$
\Pi_{\tilde{\mathrm{A}}}+\sum_{j=1}^{m} \lambda_{j} \rho_{\tilde{\mathrm{A}}}^{-1 / 2} \rho_{\tilde{\mathrm{A}}}^{(j)} \rho_{\tilde{\mathrm{A}}}^{-1 / 2}=\mathbb{1}_{\tilde{\mathrm{A}}},
$$

the suboperations $\left\{\mathcal{M}_{\tilde{\mathrm{A}} \mid \tilde{\mathrm{A}}}^{(j)}\right\}_{j=0}^{m}$ constitute an instrument.

Suppose that Alice performs this instrument, and classically communicates the outcome to Bob. If the outcome is $j$ then they perform the test with POVM element $T(j)_{\tilde{\mathrm{A}} \mathrm{B}}$ corresponding to deciding on hypothesis 0 . If their decision is stored in a register $\mathrm{C}$, then the overall measurement operation is

$$
\begin{aligned}
\mathcal{M}_{\mathrm{C} \mid \tilde{\mathrm{A}} \mathrm{B}}= & \sum_{j=0}^{m}\left\{| 0 \rangle \left\langle\left.0\right|_{\mathrm{C}} \operatorname{Tr}_{\tilde{\mathrm{A}} \mathrm{B}} T_{\tilde{\mathrm{A}} \mathrm{B}}^{(j)}\right.\right. \\
& +|1\rangle\left\langle\left. 1\right|_{\mathrm{C}} \operatorname{Tr}_{\tilde{\mathrm{A}} \mathrm{B}}\left(\mathbb{1}-T^{(j)}\right)_{\tilde{\mathrm{A}} \mathrm{B}}\right\} \mathcal{M}_{\tilde{\mathrm{A}} \mid \tilde{\mathrm{A}}}^{(j)} .
\end{aligned}
$$

Since this implementation uses only one-way classical communication followed by operations in class $\Omega$, which, by hypothesis, contains $\mathbf{L} \mathbf{C} \mathbf{1}$, this test is also in $\mathbf{T}^{\boldsymbol{\Omega}}(\tilde{\mathrm{A}}$ : B). Using $(88)$ and 83$)$, we find that the type I error of this test is

$$
\operatorname{Tr}_{\mathrm{C}}|1\rangle\left\langle\left. 1\right|_{\mathrm{C}} \mathcal{M}_{\mathrm{C} \mid \tilde{\mathrm{A}} \mathrm{B}} \mathcal{E}_{\mathrm{B} \mid \mathrm{A}}^{(0)} \rho_{\tilde{\mathrm{A}} \mathrm{A}}=\sum_{j=1}^{m} \lambda_{j} \epsilon_{j}=\epsilon\right.
$$

while its type II error is

$$
\operatorname{Tr}_{\mathrm{C}}|0\rangle\left\langle\left. 0\right|_{\mathrm{C}} \mathcal{M}_{\mathrm{C} \mid \tilde{\mathrm{A}} \mathrm{B}} \mathcal{E}_{\mathrm{B} \mid \mathrm{A}}^{(1)} \rho_{\tilde{\mathrm{A}} \mathrm{A}}=\sum_{j=1}^{m} \lambda_{j} \beta_{j},\right.
$$

and so the claimed convexity 85 does hold.

Corollary 23. $\beta_{\epsilon}^{\boldsymbol{\Omega}}\left(\mathcal{E}_{\mathrm{B} \mid \mathrm{A}}, \rho_{\mathrm{A}}\right)$ is also jointly convex in $\epsilon$ and $\rho_{\mathrm{A}}$, as it is given by maximising over functions with this property.

\section{A. Semidefinite programs}

As mentioned in the introduction, the converse for entanglement-assisted codes in terms of unrestricted hypothesis testing, and the converse for unassisted codes in terms of PPT hypothesis testing, can be formulated as semidefinite programs. Here we give the first of these in detail, and describe how to add the PPT constraint.
Letting $R_{\tilde{\mathrm{A}} \mathrm{B}}:=\rho_{\tilde{\mathrm{A}}}^{\frac{1}{2}} T_{\tilde{\mathrm{A}} \mathrm{B}} \rho_{\tilde{\mathrm{A}}}^{\frac{1}{2}}$, we have

$$
\begin{aligned}
\beta^{*}\left(T, \rho_{\tilde{\mathrm{A}}}\right) & =\left\|\operatorname{Tr}_{\mathrm{A}} R_{\tilde{\mathrm{A}} \mathrm{B}}\right\|_{\infty} \\
& =\min \left\{\lambda: \lambda \mathbb{1}_{\mathrm{B}} \geq \operatorname{Tr}_{\mathrm{A}} R_{\tilde{\mathrm{A}} \mathrm{B}}\right\},
\end{aligned}
$$

and $\alpha\left(T, \rho_{\tilde{\mathrm{A}}}\right)=\operatorname{Tr}_{\tilde{\mathrm{A}} \mathrm{B}} R_{\tilde{\mathrm{A}} \mathrm{B}} \mathcal{E}_{\mathrm{B} \mid \mathrm{A}} \Phi_{\tilde{\mathrm{A}} \mathrm{A}}$.

Proposition 24 (Primal SDP).

$$
\begin{aligned}
I_{\epsilon}^{\mathrm{ALL}}\left(\mathcal{E}_{\mathrm{B} \mid \mathrm{A}}, \rho_{\mathrm{A}}\right)= & -\log \min _{T, \lambda} \lambda \\
& \text { subject to } \\
& \operatorname{Tr}_{\mathrm{A}} R_{\tilde{\mathrm{A}} \mathrm{B}} \leq \lambda \mathbb{1}_{\mathrm{B}}, \\
& \operatorname{Tr}_{\tilde{\mathrm{A}} \mathrm{B}} R_{\tilde{\mathrm{A}} \mathrm{B}} \mathcal{E}_{\mathrm{B} \mid \mathrm{A}} \Phi_{\tilde{\mathrm{A}} \mathrm{A}} \geq 1-\epsilon, \\
& R_{\tilde{\mathrm{A}} \mathrm{B}} \leq \rho_{\tilde{\mathrm{A}}} \mathbb{1}_{\mathrm{B}}, \\
& R_{\tilde{\mathrm{A}} \mathrm{B}} \geq 0 .
\end{aligned}
$$

Since the constraints on $\rho_{\mathrm{A}}$ are semidefinite, the bound

$$
\max _{\rho_{\mathrm{A}} \in \mathcal{D}\left(\mathcal{H}_{\mathrm{A}}\right)} I_{\epsilon}^{\mathrm{ALL}}\left(\mathcal{E}_{\mathrm{B} \mid \mathrm{A}}, \rho_{\mathrm{A}}\right)
$$

from Theorem 18 is also a semidefinite program.

Remark 25. The constraint $0 \leq \mathrm{t}_{\mathrm{B} \mid \mathrm{B}} T_{\tilde{\mathrm{A}} \mathrm{B}} \leq \mathbb{1}$ (where $t_{B}$ is the transpose map on system $B$ ) is equivalent to

$$
0 \leq \mathrm{t}_{\mathrm{B} \mid \mathrm{B}} R_{\tilde{\mathrm{A}} \mathrm{B}} \leq \rho_{\tilde{\mathrm{A}}} \mathbb{1}_{\mathrm{B}} .
$$

Because the transpose map is linear, adding these constraints on $R_{\tilde{\mathrm{A}} \mathrm{B}}$ to the primal SDP above yields an SDP for $I_{\epsilon}^{\text {PPT }}\left(\mathcal{E}_{\mathrm{B} \mid \mathrm{A}}, \rho_{\mathrm{A}}\right)$.

Associating operators $F_{\tilde{\mathrm{A} B}}$ and $G_{\mathrm{B}}$ with constraints (99) and (97), and a real multiplier $\mu$ with (98) yields the Lagrangian

$$
\begin{aligned}
& \lambda+\operatorname{Tr}_{\mathrm{B}} G_{\mathrm{B}}\left(\operatorname{Tr}_{\tilde{\mathrm{A}}} R_{\tilde{\mathrm{A}} \mathrm{B}}-\lambda \mathbb{1}_{\mathrm{B}}\right) \\
+ & \operatorname{Tr}_{\tilde{\mathrm{A}} \mathrm{B}} F_{\tilde{\mathrm{A}} \mathrm{B}}\left(R_{\tilde{\mathrm{A}} \mathrm{B}}-\rho_{\tilde{\mathrm{A}}} \mathbb{1}_{\mathrm{B}}\right) \\
+ & \mu\left(1-\epsilon-\operatorname{Tr} R_{\tilde{\mathrm{A}} \mathrm{B}} \mathcal{E}_{\mathrm{B} \mid \mathrm{A}} \Phi_{\tilde{\mathrm{A}} \mathrm{A}}\right) \\
= & \operatorname{Tr}_{\tilde{\mathrm{A}} \mathrm{B}} R_{\tilde{\mathrm{A}} \mathrm{B}}\left(\mathbb{1}_{\tilde{\mathrm{A}}} G_{\mathrm{B}}+F_{\tilde{\mathrm{A}} \mathrm{B}}-\mathcal{E}_{\mathrm{B} \mid \mathrm{A}} \Phi_{\tilde{\mathrm{A}} \mathrm{A}}\right) \\
+ & \lambda\left(1-\operatorname{Tr}_{\mathrm{B}} G_{\mathrm{B}}\right)+(1-\epsilon) \mu .
\end{aligned}
$$

from which one can derive the dual SDP. Below, we show that the optimal value of this dual SDP is equal to the optimal value of the primal.

$$
\begin{gathered}
\text { Proposition } 26 \text { (Dual SDP). } \\
\qquad \begin{aligned}
I_{\epsilon}^{\mathrm{ALL}}\left(\mathcal{E}_{\mathrm{B} \mid \mathrm{A}}, \rho_{\mathrm{A}}\right)=-\log \left\{\max (1-\epsilon) \mu-\operatorname{Tr} F_{\tilde{\mathrm{A}}} \rho_{\tilde{\mathrm{A}}}\right\} \\
\text { subject to } \\
\mathbb{1}_{\tilde{\mathrm{A}}} G_{\mathrm{B}}+F_{\tilde{\mathrm{A}} \mathrm{B}} \geq \mu \mathcal{E}_{\mathrm{B} \mid \mathrm{A}} \Phi_{\tilde{\mathrm{A}} \mathrm{A}}, \\
\operatorname{Tr}_{\mathrm{B}} G_{\mathrm{B}} \leq 1, \\
G_{\mathrm{B}}, F_{\tilde{\mathrm{A}} \mathrm{B}}, \mu \geq 0 .
\end{aligned}
\end{gathered}
$$

Proof. For sufficiently large $a$, the point given by $G_{\mathrm{B}}=$ $\mathbb{1}_{\mathrm{B}} /(2 \operatorname{dim}(\mathrm{B})), F_{\tilde{\mathrm{A}} \mathrm{B}}=a \mathbb{1}_{\tilde{\mathrm{A}} \mathrm{B}}$, and any $\mu>0$ strictly satisfies the dual constraints (109 111), so the dual SDP is strictly feasible, and therefore its solution is equal to the primal solution (see Theorem 3.1 of [17]).

The maximisation of 108 over states $\rho_{\mathrm{A}}$ of $\tilde{\mathrm{A}}$ can also be formulated as an SDP, in a similar way to the primal. 


\section{B. Classical channels}

Let $\mathcal{C}_{\mathrm{A} \mid \mathrm{A}}$ and $\mathcal{C}_{\mathrm{B} \mid \mathrm{B}}$ denote the completely dephasing operations in the classical bases for $\mathrm{A}$ and $\mathrm{B}$, respectively. A channel operation $\mathcal{E}_{\mathrm{B} \mid \mathrm{A}}$ is classical if $\mathcal{E}_{\mathrm{B} \mid \mathrm{A}} \mathcal{C}_{\mathrm{A} \mid \mathrm{A}}=\mathcal{E}_{\mathrm{B} \mid \mathrm{A}}$, and $\mathcal{C}_{\mathrm{B} \mid \mathrm{B}} \mathcal{E}_{\mathrm{B} \mid \mathrm{A}}=\mathcal{E}_{\mathrm{B} \mid \mathrm{A}}$, and we can therefore restrict the minimization over average input states in our bounds to states $\rho_{\mathrm{A}}$ which are diagonal in the classical basis, thus

$$
\rho_{\mathrm{A}}=\sum_{x} p(x)|x\rangle\left\langle\left. x\right|_{\mathrm{A}} .\right.
$$

Furthermore, since the state $\mathcal{E}_{\mathrm{B} \mid \mathrm{A}} \rho_{\tilde{\mathrm{A}} \mathrm{A}}$ is invariant under the operation $\mathcal{C}_{\mathrm{B} \mid \mathrm{B}} \in \mathbf{o p s}^{\mathbf{L}}(\tilde{\mathrm{A}} \rightarrow \tilde{\mathrm{A}}, \mathrm{B} \rightarrow \mathrm{B})$, for any class $\boldsymbol{\Omega}$ containing $\mathbf{L}$ we have (by Proposition 4 )

$$
\begin{aligned}
& D_{\epsilon}^{\Omega}\left(\mathcal{E}_{\mathrm{B} \mid \mathrm{A}} \rho_{\tilde{\mathrm{A}} \mathrm{A}} \| \rho_{\tilde{\mathrm{A}}} \sigma_{\mathrm{B}}\right) \\
\geq & D_{\epsilon}^{\Omega}\left(\mathcal{C}_{\mathrm{B} \mid \mathrm{B}} \mathcal{E}_{\mathrm{B} \mid \mathrm{A}} \rho_{\tilde{\mathrm{A}} \mathrm{A}} \| \mathcal{C}_{\mathrm{B} \mid \mathrm{B}} \rho_{\tilde{\mathrm{A}}} \sigma_{\mathrm{B}}\right) \\
= & D_{\epsilon}^{\Omega}\left(\mathcal{E}_{\mathrm{B} \mid \mathrm{A}} \rho_{\tilde{\mathrm{A}} \mathrm{A}} \| \rho_{\tilde{\mathrm{A}}} \sigma_{\mathrm{B}}^{\prime}\right)
\end{aligned}
$$

where $\sigma_{\mathrm{B}}^{\prime}$ is diagonal in the classical basis of B. Therefore, we can also restrict the optimisation over $\sigma_{\mathrm{B}}$ to classical states and, by the discussion in subsection IIB, restrict the test POVM element to be diagonal in the classical basis. Therefore, Theorems 18 and 19 both reduce to the PPV converse for finite alphabets when the channel is classical.

\section{Comparison with Wang-Renner}

In our notation, the Wang-Renner converse states that for c-q channels with finite input alphabet $A$ and output states $\tau(x)_{\mathrm{B}}$ for $x \in \mathrm{A}$

$$
\log M_{\epsilon} \leq \sup _{p} D_{\epsilon}^{\mathbf{A L L}}\left(\tau_{\mathrm{CB}} \| \tau_{\mathrm{C}} \tau_{\mathrm{B}}\right)
$$

where $\mathrm{C}$ is a system of dimension $|\mathrm{A}|$ and $\tau_{\mathrm{CB}}:=$ $\sum_{x \in \mathrm{A}} p(x)|x\rangle\left\langle\left. x\right|_{\mathrm{C}} \otimes \tau(x)_{\mathrm{B}}\right.$. To apply their converse to general channels, one notes that any (unassisted) code for a general quantum channel, induces a c-q channel by its specification of the input states used in the code. Together with the choice of $p$ the yields a c-q state

$$
\tau_{\mathrm{CB}}=\sum_{x} p(x)|x\rangle\left\langle\left. x\right|_{\mathrm{C}} \otimes \mathcal{E}_{\mathrm{B} \mid \mathrm{A}}\left[\rho(x)_{\mathrm{A}}\right]\right.
$$

Optimising the Wang-Renner bound over all choices of input states and distributions $p$ such that the average channel input to the quantum channel is $\rho_{\mathrm{A}}$, one obtains

$$
M_{\epsilon}\left(\mathcal{E}_{\mathrm{B} \mid \mathrm{A}}\right) \leq \chi_{\epsilon}\left(\mathcal{E}_{\mathrm{B} \mid \mathrm{A}}, \rho_{\mathrm{A}}\right)
$$

where

\section{Definition 27.}

$\chi_{\epsilon}\left(\mathcal{E}_{\mathrm{B} \mid \mathrm{A}}, \rho_{\mathrm{A}}\right):=\max _{\eta_{\mathrm{CA}} \in \operatorname{Ens}\left(\rho_{\mathrm{A}}\right)} D_{\epsilon}^{\mathrm{ALL}}\left(\mathcal{E}_{\mathrm{B} \mid \mathrm{A}} \eta_{\mathrm{CA}} \| \eta_{\mathrm{C}} \mathcal{E}_{\mathrm{B} \mid \mathrm{A}} \rho_{\mathrm{A}}\right)$, where $\operatorname{Ens}\left(\rho_{\mathrm{A}}\right)$ be the set of all states of the form

$$
\sum_{k=1}^{\operatorname{dim}(\mathrm{C})} p_{k}|k\rangle\left\langle\left. k\right|_{\mathrm{C}} \otimes \rho(k)_{\mathrm{A}}\right.
$$

where $\mathrm{C}$ is some finite dimensional system (acting as a classical register) and where $p_{k} \geq 0, \sum_{k} p_{k}=1, \rho(k)_{\mathrm{A}} \in$ states $(\mathrm{A})$, and $\sum_{k} p_{k} \rho(k)_{\mathrm{A}}=\rho_{\mathrm{A}}$.

This notation is motivated by the fact that the Holevo bound [18] is given by $\chi\left(\mathcal{E}_{\mathrm{B} \mid \mathrm{A}}, \rho_{\mathrm{A}}\right)=$ $\max _{\eta_{\mathrm{CA}} \in \operatorname{Ens}\left(\rho_{\mathrm{A}}\right)} D\left(\mathcal{E}_{\mathrm{B} \mid \mathrm{A}} \eta_{\mathrm{CA}} \| \eta_{\mathrm{C}} \mathcal{E}_{\mathrm{B} \mid \mathrm{A}} \rho_{\mathrm{A}}\right)$, where $D$ is the usual quantum relative entropy [19].

\section{Proposition 28.}

$$
\chi_{\epsilon}\left(\mathcal{E}_{\mathrm{B} \mid \mathrm{A}}, \rho_{\mathrm{A}}\right)=D_{\epsilon}^{\mathrm{LC} 1}\left(\mathcal{E}_{\mathrm{B} \mid \mathrm{A}} \rho_{\tilde{\mathrm{A}} \mathrm{A}} \| \rho_{\tilde{\mathrm{A}}} \mathcal{E}_{\mathrm{B} \mid \mathrm{A}} \rho_{\mathrm{A}}\right)
$$

Proof. Let Alice's measurement have the POVM elements $F(k)_{\tilde{A}}$ where $k$ labels the outcome which she sends to Bob. There is no loss of generality in having Alice perform her measurement before Bob does anything, and storing the outcome in a classical register $\mathrm{C}$ to which Bob has access.

Let $p(k):=\operatorname{Tr}_{\tilde{\tilde{A}}} F(k)_{\tilde{\mathrm{A}}}$ be the probability of outcome $k$ and $\rho(k)_{\mathrm{A}}=\operatorname{Tr}_{\tilde{\mathrm{A}}}\left(\rho_{\mathrm{A}}^{\frac{1}{2}} \Phi_{\tilde{\mathrm{A}} \mathrm{A}} \rho_{\mathrm{A}}^{\frac{1}{2}}\right) F_{\tilde{\mathrm{A}}}(k)$. Under hypothesis 0 the state of $\mathrm{CB}$ is $\mathcal{E}_{\mathrm{B} \mid \mathrm{A}} \eta_{\mathrm{CA}}$, where

$$
\eta_{\mathrm{CA}}=\sum_{k} p(k)|k\rangle\left\langle\left. k\right|_{\mathrm{C}} \otimes \rho(k)_{\mathrm{A}},\right.
$$

while under hypothesis 1 the state is $\eta_{\mathrm{C}} \mathcal{E}_{\mathrm{B} \mid \mathrm{A}} \eta_{\mathrm{A}}$. Clearly $\eta_{\mathrm{A}}=\sum_{k} p(k) \rho(k)_{\mathrm{A}}=\rho_{\mathrm{A}}$. Therefore,

$$
\begin{aligned}
& D_{\epsilon}^{\mathbf{L C} 1}\left(\mathcal{E}_{\mathrm{B} \mid \mathrm{A}} \rho_{\tilde{\mathrm{A}} \mathrm{A}} \| \rho_{\tilde{\mathrm{A}}} \mathcal{E}_{\mathrm{B} \mid \mathrm{A}} \rho_{\mathrm{A}}\right) \\
= & \max _{\eta_{\mathrm{CA}} \in \operatorname{Ens}\left(\rho_{\mathrm{A}}\right)} D_{\epsilon}^{\mathbf{A L L}}\left(\mathcal{E}_{\mathrm{B} \mid \mathrm{A}} \eta_{\mathrm{CA}} \| \eta_{\mathrm{C}} \mathcal{E}_{\mathrm{B} \mid \mathrm{A}} \rho_{\mathrm{A}}\right) \\
= & \chi_{\epsilon}\left(\mathcal{E}_{\mathrm{B} \mid \mathrm{A}}, \rho_{\mathrm{A}}\right) .
\end{aligned}
$$

Corollary 29. From the above proposition and the definitions of the quantities involved, the inequalities

$$
I_{\epsilon}^{\mathrm{L}}\left(\mathcal{E}_{\mathrm{B} \mid \mathrm{A}}, \rho_{\mathrm{A}}\right) \leq I_{\epsilon}^{\mathrm{LC} 1}\left(\mathcal{E}_{\mathrm{B} \mid \mathrm{A}}, \rho_{\mathrm{A}}\right) \leq \chi_{\epsilon}\left(\mathcal{E}_{\mathrm{B} \mid \mathrm{A}}, \rho_{\mathrm{A}}\right)
$$

follow immediately.

\section{Asymptotics}

It was already noted in 2 that the (asymptotically tight) Holevo bound on unassisted codes can be recovered from an asymptotic analysis of the Wang-Renner bound, which our bounds on unassisted codes subsume (in fact, an argument of [5] can be used to show that the converse part of the HSW theorem [20, 21] can also be derived). 
For entanglement-assisted coding over memoryless quantum channels, Shannon's noisy channel coding theorem has a beautiful generalisation due to Bennett, Shor, Smolin and Thapliyal:

$$
\lim _{\epsilon \rightarrow 0} \lim _{n \rightarrow \infty} \frac{1}{n} \log M_{\epsilon}^{\mathrm{E}}\left(\left(\mathcal{E}^{\otimes n}\right)_{\mathrm{B}^{n} \mid \mathrm{A}^{n}}\right)=\max _{\rho_{\mathrm{A}}} I\left(\mathcal{E}_{\mathrm{B} \mid \mathrm{A}}, \rho_{\mathrm{A}}\right)
$$

where

$$
I\left(\mathcal{E}_{\mathrm{B} \mid \mathrm{A}}, \rho_{\mathrm{A}}\right):=S\left(\rho_{\mathrm{A}}\right)+S\left(\mathcal{E}_{\mathrm{B} \mid \mathrm{A}}\left(\rho_{\mathrm{A}}\right)\right)-S\left(\mathcal{E}_{\mathrm{B} \mid \mathrm{A}} \rho_{\tilde{\mathrm{A}}}\right)
$$

is the quantum mutual information between systems $\tilde{\mathrm{A}}$ and $\mathrm{B}$ when the state of $\tilde{\mathrm{AB}}$ is $\mathcal{E}_{\mathrm{B} \mid \mathrm{A}} \rho_{\tilde{\mathrm{A}} \mathrm{A}}$. As noted, for classical channels Theorem 18 reduces to Theorem 27 of [6]. In section III.G of [6] it is shown how to derive a Fano-type converse from their Theorem 27. The derivation and result generalise perfectly to the entanglementassisted codes for quantum channels: As usual, the binary entropy is $h(p):=-(1-p) \log (1-p)-p \log p$, and the binary relative entropy is

$$
\begin{aligned}
d(p \| q): & =D((p, 1-p) \|(q, 1-q)) \\
& =p \log \frac{p}{q}+(1-p) \log \frac{1-p}{1-q} \\
& \geq p \log \frac{1}{q}-h(p) .
\end{aligned}
$$

By the data processing inequality for quantum relative entropy under CPTP maps, and 131

$$
\begin{aligned}
D\left(\rho_{0} \| \rho_{1}\right) & \geq d\left(1-\epsilon \| \beta_{\epsilon}\left(\rho_{0}, \rho_{1}\right)\right) \\
& \geq(1-\epsilon) \log \frac{1}{\beta_{\epsilon}\left(\rho_{0}, \rho_{1}\right)}-h(\epsilon) .
\end{aligned}
$$

Therefore,

$$
D_{\epsilon}^{\mathbf{A L L}}\left(\rho_{0} \| \rho_{1}\right) \leq\left(D\left(\rho_{0} \| \rho_{1}\right)+h(\epsilon)\right) /(1-\epsilon) .
$$

Setting $\rho_{0}=\mathcal{E}_{\mathrm{B} \mid \mathrm{A}} \rho_{\tilde{\mathrm{A}} \mathrm{A}}$ and $\rho_{1}=\rho_{\tilde{\mathrm{A}}} \sigma_{\mathrm{B}}$, and minimizing over $\sigma_{\mathrm{B}}$ yields

\section{Lemma 30.}

$$
I_{\epsilon}^{\mathbf{A L L}}\left(\mathcal{E}_{\mathrm{B} \mid \mathrm{A}}, \rho_{\mathrm{A}}\right) \leq\left(I\left(\mathcal{E}_{\mathrm{B} \mid \mathrm{A}}, \rho_{\mathrm{A}}\right)+h(\epsilon)\right) /(1-\epsilon)
$$

The converse part of this theorem is easily derived from the previous lemma, which tells us that

$$
\begin{aligned}
& \log M_{\epsilon}^{\mathrm{E}}\left(\left(\mathcal{E}^{\otimes n}\right)_{\left.\mathrm{B}^{n} \mid \mathrm{A}^{n}, \rho_{\mathrm{A}^{n}}\right)}\right. \\
\leq & \max _{\rho_{\mathrm{A}}} \frac{I\left(\left(\mathcal{E}^{\otimes n}\right)_{\mathrm{B}^{n}} \mid \mathrm{A}^{n}, \rho_{\mathrm{A}^{n}}\right)+h(\epsilon)}{1-\epsilon} .
\end{aligned}
$$

In 22] Adami and Cerf show that

$$
I\left(\mathcal{E}_{\mathrm{B}_{1} \mid \mathrm{A}_{1}}^{(1)} \mathcal{E}_{\mathrm{B}_{2} \mid \mathrm{A}_{2}}^{(2)}, \rho_{\mathrm{A}_{1} \mathrm{~A}_{2}}\right)=I\left(\mathcal{E}_{\mathrm{B}_{1} \mid \mathrm{A}_{1}}^{(1)}, \rho_{\mathrm{A}_{1}}\right)+I\left(\mathcal{E}_{\mathrm{B}_{2} \mid \mathrm{A}_{2}}^{(2)}, \rho_{\mathrm{A}_{2}}\right),
$$

so we have

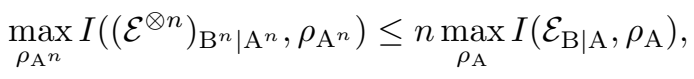

and

$$
\lim _{n \rightarrow \infty} \frac{1}{n} \log M_{\epsilon}\left(\left(\mathcal{E}^{\otimes n}\right)_{\mathrm{B}^{n} \mid \mathrm{A}^{n}}\right) \leq \frac{1}{1-\epsilon} \max _{\rho_{\mathrm{A}}} I\left(\mathcal{E}_{\mathrm{B} \mid \mathrm{A}}, \rho_{\mathrm{A}}\right) .
$$

Taking the limit $\epsilon \rightarrow 0$ completes the proof.

\section{E. Using symmetries}

We now show how symmetries of $\mathcal{E}_{\mathrm{B} \mid \mathrm{A}}$ can be used to simplify the computation of $I_{\epsilon}^{\boldsymbol{\Omega}}\left(\mathcal{E}_{\mathrm{B} \mid \mathrm{A}}, \rho_{\mathrm{A}}\right)$. For classical channels, analogous results were obtained in [7] (but note that 7] also deals with infinite input/output alphabets) and similar ideas were discussed in 13 .

Suppose that there is a group $G$ with a representation $g \mapsto g_{\mathrm{A} \mid \mathrm{A}} \in \operatorname{ops}(\mathrm{A} \rightarrow \mathrm{A})$ for all $g \in G$, given by

$$
g_{\mathrm{A} \mid \mathrm{A}} \tau_{\mathrm{A}}=U(g)_{\mathrm{A}} \tau_{\mathrm{A}} U(g)_{\mathrm{A}}^{\dagger},
$$

and a representation $g \mapsto g_{\mathrm{B} \mid \mathrm{B}} \in \operatorname{ops}(\mathrm{B} \rightarrow \mathrm{B})$, given by

$$
g_{\mathrm{B} \mid \mathrm{B}} \tau_{\mathrm{B}}=V(g)_{\mathrm{B}} \tau_{\mathrm{B}} V(g)_{\mathrm{B}}^{\dagger},
$$

where $U$ and $V$ are unitary representations of $G$, and let

$$
g_{\tilde{\mathrm{A}} \mid \tilde{\mathrm{A}}} \tau_{\tilde{\mathrm{A}}}:=U(g)_{\mathrm{A}}^{*} \tau_{\tilde{\mathrm{A}}} U(g)_{\mathrm{A}}^{\mathrm{T}} .
$$

Suppose that $\mathcal{E}_{\mathrm{B} \mid \mathrm{A}}$ possesses the $G$-covariance

$$
\forall g \in G: \mathcal{E}_{\mathrm{B} \mid \mathrm{A}} g_{\mathrm{A} \mid \mathrm{A}}=g_{\mathrm{B} \mid \mathrm{B}} \mathcal{E}_{\mathrm{B} \mid \mathrm{A}} .
$$

Proposition 31. For any $\Omega \supseteq \mathbf{L}$ and for all $g \in G$,

$$
\begin{aligned}
& D_{\epsilon}^{\boldsymbol{\Omega}}\left(\mathcal{E}_{\mathrm{B} \mid \mathrm{A}} g_{\tilde{\mathrm{A}} \mid \tilde{\mathrm{A}}} g_{\mathrm{A} \mid \mathrm{A}} \rho_{\tilde{\mathrm{A}} \mathrm{A}} \| g_{\tilde{\mathrm{A}} \mid \tilde{\mathrm{A}}} g_{\mathrm{B} \mid \mathrm{B}} \rho_{\tilde{\mathrm{A}}} \sigma_{\mathrm{B}}\right) \\
= & D_{\epsilon}^{\boldsymbol{\Omega}}\left(\mathcal{E}_{\mathrm{B} \mid \mathrm{A}} \rho_{\tilde{\mathrm{A}} \mathrm{A}} \| \rho_{\tilde{\mathrm{A}}} \sigma_{\mathrm{B}}\right)
\end{aligned}
$$

and $I_{\epsilon}^{\boldsymbol{\Omega}}\left(\mathcal{E}_{\mathrm{B} \mid \mathrm{A}}, g_{\mathrm{A} \mid \mathrm{A}} \rho_{\mathrm{A}}\right)=I_{\epsilon}^{\boldsymbol{\Omega}}\left(\mathcal{E}_{\mathrm{B} \mid \mathrm{A}}, \rho_{\mathrm{A}}\right)$.

Proof. The first claim follows from

$$
\begin{aligned}
& \mathcal{E}_{\mathrm{B} \mid \mathrm{A}} g_{\mathrm{A} \mid \mathrm{A}} \rho_{\tilde{\mathrm{A}} \mathrm{A}} \\
= & \mathcal{E}_{\mathrm{B} \mid \mathrm{A}}\left[U(g)_{\mathrm{A}} \rho_{\mathrm{A}}^{\frac{1}{2}} U(g)_{\mathrm{A}}^{\dagger} \Phi_{\tilde{\mathrm{A} A}} U(g)_{\mathrm{A}} \rho_{\mathrm{A}}^{\frac{1}{2}} U(g)_{\mathrm{A}}^{\dagger}\right] \\
= & \mathcal{E}_{\mathrm{B} \mid \mathrm{A}}\left[U(g)_{\mathrm{A}} \rho_{\mathrm{A}}^{\frac{1}{2}} U(g)_{\tilde{\mathrm{A}}}^{*} \Phi_{\tilde{\mathrm{A} A}} U(g)_{\tilde{\mathrm{A}}}^{\mathrm{T}} \rho_{\mathrm{A}}^{\frac{1}{2}} U(g)_{\mathrm{A}}^{\dagger}\right] \\
= & V(g)_{\mathrm{B}} U(g)_{\tilde{\mathrm{A}}}^{*} \mathcal{E}_{\mathrm{B} \mid \mathrm{A}}\left[\rho_{\mathrm{A}}^{\frac{1}{2}} \Phi_{\tilde{\mathrm{A}} \mathrm{A}} \rho_{\mathrm{A}}^{\frac{1}{2}}\right] V(g)_{\mathrm{B}} U(g)_{\tilde{\mathrm{A}}}^{\mathrm{T}} \\
= & g_{\tilde{\mathrm{A}} \mid \tilde{\mathrm{A}}} g_{\mathrm{B} \mid \mathrm{B}} \mathcal{E}_{\mathrm{B} \mid \mathrm{A}} \rho_{\tilde{\mathrm{A}} \mathrm{A}},
\end{aligned}
$$

the fact that $g_{\tilde{\mathrm{A}} \mid \tilde{\mathrm{A}}} g_{\mathrm{B} \mid \mathrm{B}}$ and its inverse belong to $\mathbf{L}(\tilde{\mathrm{A}} \rightarrow$ $\tilde{\mathrm{A}}, \mathrm{B} \rightarrow \mathrm{B}) \subseteq \Omega$, and Corollary 5 . We use this to prove the second claim thus:

$$
\begin{aligned}
& I_{\epsilon}^{\boldsymbol{\Omega}}\left(\mathcal{E}_{\mathrm{B} \mid \mathrm{A}}, \rho_{\mathrm{A}}\right) \\
= & \min _{\sigma_{\mathrm{B}}} D_{\epsilon}^{\boldsymbol{\Omega}}\left(\mathcal{E}_{\mathrm{B} \mid \mathrm{A}} \rho_{\tilde{\mathrm{AA}}} \| \rho_{\tilde{\mathrm{A}}} \sigma_{\mathrm{B}}\right) \\
= & D_{\epsilon}^{\boldsymbol{\Omega}}\left(\mathcal{E}_{\mathrm{B} \mid \mathrm{A}} \rho_{\tilde{\mathrm{AA}}} \| \rho_{\tilde{\mathrm{A}}} \sigma_{\mathrm{B}}^{0}\right) \\
= & D_{\epsilon}^{\boldsymbol{\Omega}}\left(\mathcal{E}_{\mathrm{B} \mid \mathrm{A}} g_{\tilde{\mathrm{A}} \mid \tilde{\mathrm{A}}} g_{\mathrm{A} \mid \mathrm{A}} \rho_{\tilde{\mathrm{A}} \mathrm{A}} \| g_{\tilde{\mathrm{A}} \mid \tilde{\mathrm{A}}} g_{\mathrm{B} \mid \mathrm{B}} \rho_{\tilde{\mathrm{A}}} \sigma_{\mathrm{B}}^{0}\right) \\
\geq & \min _{\sigma_{\mathrm{B}}} D_{\epsilon}^{\boldsymbol{\Omega}}\left(\mathcal{E}_{\mathrm{B} \mid \mathrm{A}} g_{\tilde{\mathrm{A}} \mid \tilde{\mathrm{A}}} g_{\mathrm{A} \mid \mathrm{A}} \rho_{\tilde{\mathrm{A}} \mathrm{A}} \| g_{\tilde{\mathrm{A}} \mid \tilde{\mathrm{A}}} \rho_{\tilde{\mathrm{A}}} \sigma_{\mathrm{B}}\right) \\
= & I_{\epsilon}^{\boldsymbol{\Omega}}\left(\mathcal{E}_{\mathrm{B} \mid \mathrm{A}}, g_{\mathrm{A} \mid \mathrm{A}} \rho_{\mathrm{A}}\right) .
\end{aligned}
$$


Since $g$ has an inverse in $G$, the reverse inequality holds too.

Suppose that there is a Haar ( $G$-invariant) measure $\mu$ on $G$, and let $\bar{\rho}_{\mathrm{A}}:=\int_{G} d \mu(g) g_{\mathrm{A} \mid \mathrm{A}} \rho_{\mathrm{A}}$. Then, $\bar{\rho}_{\mathrm{A}}$ is invariant under the action $g_{\mathrm{A} \mid \mathrm{A}}$, and by Jensen's inequality, Corollary 23, and Proposition 31.

$$
\begin{aligned}
\beta_{\epsilon}^{\boldsymbol{\Omega}}\left(\mathcal{E}_{\mathrm{B} \mid \mathrm{A}}, \bar{\rho}_{\mathrm{A}}\right) & \leq \int_{G} d \mu(g) \beta_{\epsilon}^{\boldsymbol{\Omega}}\left(\mathcal{E}_{\mathrm{B} \mid \mathrm{A}}, g_{\mathrm{A} \mid \mathrm{A}} \rho_{\mathrm{A}}\right) \\
& =\beta_{\epsilon}^{\boldsymbol{\Omega}}\left(\mathcal{E}_{\mathrm{B} \mid \mathrm{A}}, \rho_{\mathrm{A}}\right) .
\end{aligned}
$$

Therefore, the optimisation over $\rho_{\mathrm{A}}$ can be restricted to those density operators invariant under the action of $g_{\mathrm{A} \mid \mathrm{A}}$.

An important type of symmetry that an operation representing $n$ channel uses may possess is permutation covariance. For example, this applies to $n$ uses of a memoryless channel.

For any element $\pi$ of the symmetric group $S_{n}$, and $n$-partite system $\mathrm{Q}^{n}:=\mathrm{Q}_{1} \mathrm{Q}_{2} \ldots \mathrm{Q}_{n}$ consisting of $n$ isomorphic systems $\mathrm{Q}_{j}$, let $\pi_{\mathrm{Q}^{n} \mid \mathrm{Q}^{n}} \in \operatorname{ops}\left(\mathrm{Q}^{n} \rightarrow \mathrm{Q}^{n}\right)$ denote the unitary operation which permutes the $n$ systems.

An operation $\mathcal{E}_{\mathrm{B}^{n} \mid \mathrm{A}^{n}} \in \mathbf{o p s}\left(\mathrm{A}^{n} \rightarrow \mathrm{B}^{n}\right)$ is permutation covariant if

$$
\mathcal{E}_{\mathrm{B}^{n} \mid \mathrm{A}^{n}} \pi_{\mathrm{A}^{n} \mid \mathrm{A}^{n}}=\pi_{\mathrm{B}^{n} \mid \mathrm{B}^{n}} \mathcal{E}_{\mathrm{B}^{n} \mid \mathrm{A}^{n}} .
$$

Suppose that, in addition to permutation invariance of the $n$ uses, each use of the channel is $G$-covariant in the sense that

$$
\mathcal{E}_{\mathrm{B}^{n} \mid \mathrm{A}^{n}} g_{\mathrm{A}_{j} \mid \mathrm{A}_{j}}=g_{\mathrm{B}_{j} \mid \mathrm{B}_{j}} \mathcal{E}_{\mathrm{B}^{n} \mid \mathrm{A}^{n}}
$$

for all $g \in G$ and $j \in\{1, \ldots, n\}$. Here the representations of $G$ on each system are the same, except that they act on different systems. This is the case, for example, if $\mathcal{E}_{\mathrm{B}^{n} \mid \mathrm{A}^{n}}$ is $n$ uses of a $G$-covariant memoryless channel.

To every ordered pair $(\pi, \mathbf{g})$ where $\pi \in S_{n}$ and $\mathbf{g} \in G^{\times n}$, we can associate an action $(\pi, \mathbf{g})_{\mathrm{A}^{n} \mid \mathrm{A}^{n}}:=$ $\pi_{\mathrm{A}^{n} \mid \mathrm{A}^{n}} \mathbf{g}_{\mathrm{A}^{n} \mid \mathrm{A}^{n}}$. Here the action on $\mathrm{A}^{n}$ for $\mathbf{g}=$ $\left(g^{(1)}, \ldots, g^{(n)}\right)$ is $\mathbf{g}_{\mathrm{A}^{n} \mid \mathrm{A}^{n}}:=\bigotimes_{j=1}^{n} g_{\mathrm{A}_{j} \mid \mathrm{A}_{j}}^{(j)}$ and $\mathbf{g}_{\mathrm{B}^{n} \mid \mathrm{B}^{n}}:=$ $\bigotimes_{j=1}^{n} g_{\mathrm{B}_{j} \mid \mathrm{B}_{j}}^{(j)}$. Under composition these actions constitute a group, which is a semi-direct product of $G^{\times n}$ and $S_{n}\left(G^{\times n}\right.$ being the normal subgroup) which we denote $S_{n} \ltimes G^{\times n}$. Defining the action of $(\pi, \mathbf{g}) \in S_{n} \ltimes G^{\times n}$ on states of $\mathrm{B}^{n}$ by $(\pi, \mathbf{g})_{\mathrm{B}^{n} \mid \mathrm{B}^{n}}:=\pi_{\mathrm{B}^{n} \mid \mathrm{B}^{n}} \mathbf{g}_{\mathrm{B}^{n} \mid \mathrm{B}^{n}}$, we have

$$
\mathcal{E}_{\mathrm{B}^{n} \mid \mathrm{A}^{n}}(\pi, \mathbf{g})_{\mathrm{A}^{n} \mid \mathrm{A}^{n}}=(\pi, \mathbf{g})_{\mathrm{B}^{n} \mid \mathrm{B}^{n}} \mathcal{E}_{\mathrm{B}^{n} \mid \mathrm{A}^{n}} .
$$

\section{EXAMPLE: THE DEPOLARISING CHANNEL}

A single use of the $d$-dimensional depolarising channel with parameter $p$ and $d$-dimensional input and output systems A and B has the operation

$$
\mathcal{D}_{\mathrm{B} \mid \mathrm{A}} \tau_{\mathrm{A}}=(1-p) \tau_{\mathrm{B}}+p \operatorname{Tr}\left(\tau_{\mathrm{B}}\right) \mu_{\mathrm{B}},
$$

where, for any system $Q, \mu_{\mathrm{Q}}:=\mathbb{1}_{\mathrm{Q}} / \operatorname{dim}(\mathrm{Q})$ denotes the maximally mixed state on that system. For $n$ uses the operation is

$$
\mathcal{D}_{\mathrm{B}^{n} \mid \mathrm{A}^{n}}^{\otimes n}=\mathcal{D}_{\mathrm{B}_{1} \mid \mathrm{A}_{1}} \ldots \mathcal{D}_{\mathrm{B}_{n} \mid \mathrm{A}_{n}}
$$

which has the covariance group $S_{n} \ltimes \mathrm{U}(d)^{\times n}$.

The only input and output states with the corresponding invariances are the maximally mixed states. Therefore,

$$
\sup _{\rho_{\mathrm{A}^{n}}} I_{\epsilon}^{\mathbf{A L L}}\left(\mathcal{D}_{\mathrm{B}^{n} \mid \mathrm{A}^{n}}^{\otimes n}, \rho_{\mathrm{A}^{n}}\right)=D_{\epsilon}^{\mathbf{A L L}}\left(\phi(p)_{\tilde{\mathrm{A}}^{n} \mathrm{~B}^{n}}^{\otimes n} \| \mu_{\tilde{\mathrm{A}}^{n}} \mu_{\mathrm{B}^{n}}\right)
$$

where $\phi(p)_{\tilde{\mathrm{A}} \mathrm{B}}:=\mathcal{D}_{\mathrm{B} \mid \mathrm{A}}\left[\Phi_{\tilde{\mathrm{AA}}} / d\right]=(1-p) \Phi_{\tilde{\mathrm{A}}} / d+p \mu_{\tilde{\mathrm{A}}} \mu_{\mathrm{B}}$ is an isotropic state. Since the arguments of $D_{\epsilon}^{\mathbf{A L L}}$ commute in this expression, this is equivalent to a classical hypothesis test between the distributions given by the eigenvalues of the two states. In fact, the degeneracy of the eigenvalues makes it is equivalent to deciding between hypotheses on the distribution of $n$ samples of a binary variable: Hypothesis $H_{0}$ is that the samples are drawn i.i.d. with probability $(1-p)+p / d^{2}$ of being 0 , and hypothesis $H_{1}$ is that the samples are drawn i.i.d. with probability $1 / d^{2}$ of being 0 . Therefore,

$$
\begin{aligned}
& D_{\epsilon}^{\mathbf{A L L}}\left(\phi(p)_{\tilde{\mathrm{A}}^{n} \mathrm{~B}^{n}}^{\otimes n} \| \mu_{\tilde{\mathrm{A}}^{n}} \mu_{\mathrm{B}^{n}}\right) \\
= & D_{\epsilon}^{\mathbf{A L L}}\left((\mu, 1-\mu)^{\otimes n},(\lambda, 1-\lambda)^{\otimes n}\right)
\end{aligned}
$$

where $\mu=(1-p)+p / d^{2}$ and $\lambda=1 / d^{2}$, and Proposition 32 gives a formula for this quantity which is easy to evaluate exactly (as we have done for Fig. 4).

Proposition 32. Let $\mu \geq \lambda$ be two probabilities.

$$
\beta_{\epsilon}\left((\mu, 1-\mu)^{\otimes n},(\lambda, 1-\lambda)^{\otimes n}\right)=(1-\gamma) \beta_{\ell(\epsilon)}+\gamma \beta_{\ell(\epsilon)+1}
$$

where

$$
\begin{aligned}
& \alpha_{\ell}=\sum_{j=0}^{\ell-1}\left(\begin{array}{l}
n \\
j
\end{array}\right) \mu^{j}(1-\mu)^{n-j}, \\
& \beta_{\ell}=\sum_{j=l}^{n}\left(\begin{array}{l}
n \\
j
\end{array}\right) \lambda^{j}(1-\lambda)^{n-j},
\end{aligned}
$$

$\ell(\epsilon)$ is the value of $l$ satisfying $\alpha_{\ell} \leq \epsilon \leq \alpha_{\ell+1}$ and $\gamma=$ $\left(\alpha-\alpha_{\ell(\alpha)}\right) /\left(\alpha_{\ell(\alpha)+1}-\alpha_{\ell(\alpha)}\right)$.

Proof. This is just optimising over the optimal (classical) hypothesis tests identified by the Neyman-Pearson lemma. The same expression is given in [6].

\section{CONVERSES AND CRYPTOGRAPHY}

In 23] a strong converse was proven for the classical capacity of many quantum channels $\mathcal{N}$, including depolarising noise. The results of [23] had a nice application 


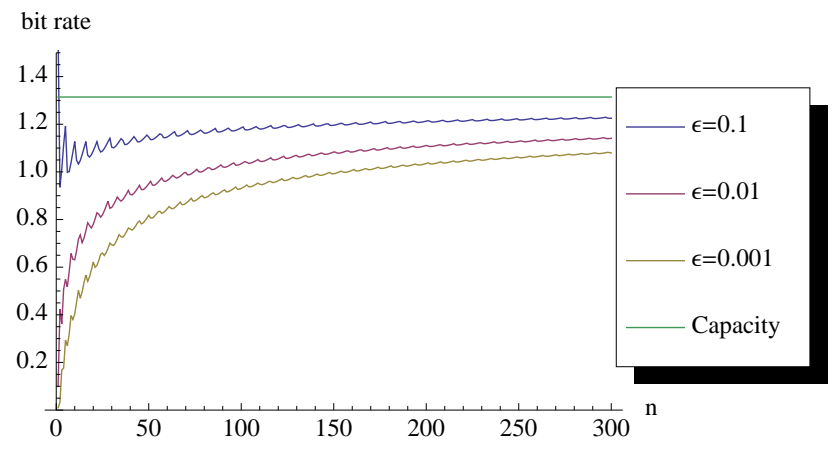

FIG. 4: Our upper bound (Theorem 18) on the rate of entanglement-assisted codes evaluated (see Section VI) for three different error probabilities $\epsilon$, for the qubit depolarising channel with failure probability 0.15 . The red line marks the capacity of the channel (roughly 1.31 bits/channel use) as given by the formula of Bennett, Shor, Smolin and Thapliyal [12].

to proving security in so-called noisy-storage model of quantum cryptography 24]. This model allows for the secure implementation of any two-party cryptographic task under the assumption that the adversary's quantum memory is noisy. Examples of such tasks include bit commitment and oblivious transfer, which are impossible to achieve without assumptions [25, 26].

Concretely, the noisy-storage model assumes that during waiting times introduced into the protocol, the adversary can only store quantum information in a memory device modelled by a channel $\mathcal{E}_{\mathrm{B} \mid \mathrm{A}}$. Otherwise the adversary is all powerful and may even use a quantum computer to peform the most advantageous error-correcting encoding. At the beginning of the waiting time, the state of the protocol can be described as a cq-state $\rho_{\mathrm{XKA}}$ where $\mathrm{X}$ is a classical register held by the honest party and storing a string $X$ and $\mathrm{A}$ and $\mathrm{K}$ are the quantum and classical registers of the dishonest party respectively. After the wait time, the state of the protocol is described by $\mathcal{E}_{\mathrm{B} \mid \mathrm{A}} \rho_{\mathrm{XKA}}$. The security of all protocols proposed in this model requires a bound on the min-entropy $\mathrm{H}_{\text {min }}(\mathrm{X} \mid \mathrm{BK})=-\log P_{\text {guess }}(\mathrm{X} \mid \mathrm{BK})$, where $P_{\text {guess }}(\mathrm{X} \mid \mathrm{BK})$ is the probability that the adversary holding $\mathrm{B}$ and $\mathrm{K}$ manages to guess $X$ maximized over all possible measurements on BK. Bounds on this quantity can be linked to the classical capacity [24, the entanglement cost 27] or the quantum capacity [28] of $\mathcal{E}_{\mathrm{B} \mid \mathrm{A}}$. Yet, explicit bounds on the min-entropy which would enable practical implementations of such protocols [29, 30] are elusive. In particular, up to this date no statements are known for arbitrary channels $\mathcal{E}_{\mathrm{B} \mid \mathrm{A}}$, such as channels which do not obey a strong converse or simply structureless channels. Our method can be used to compute explicit bounds on the min-entropy for arbitrary channels.

The key to relating our analysis to a study of the min- entropy is the observation [24] that

$$
\begin{aligned}
& \mathrm{H}_{\min }(\mathrm{X} \mid \mathrm{BK}) \geq \\
& \quad-\log \max _{\mathcal{Z}} \operatorname{Pr}\left(\hat{W}=W \mid \mathcal{E}_{\mathrm{B} \mid \mathrm{A}}, \mathcal{Z}, S_{\left\lfloor\mathrm{H}_{\min }(\mathrm{X} \mid \mathrm{K})\right\rfloor}\right) .
\end{aligned}
$$

That is, by understanding the adversary's knowledge $\mathrm{H}_{\text {min }}(\mathrm{X} \mid \mathrm{K})$ conditioned on the classical knowledge $\mathrm{K}$ alone, and the properties of the channel $\mathcal{E}_{\mathrm{B} \mid \mathrm{A}}$ we can bound the adversary's knowledge about a string $\mathrm{X}$ given both $\mathrm{B}$ and $\mathrm{K}$. In 24 the bound $\mathrm{H}_{\min }(\mathrm{X} \mid \mathrm{K}) \gtrsim n / 2$ was obtained from an uncertainty relation for BB84 measurements that was used to generate the $n$-bit string $X$. Different measurements lead to higher (or lower) values of

$$
\frac{\mathrm{H}_{\min }(\mathrm{X} \mid \mathrm{K})}{n}=: \hat{R} \text {. }
$$

In 24 , a strong converse for some channels $\mathcal{N}^{\otimes \ell}$ was then used to bound the r.h.s. for $\mathcal{E}_{\mathrm{B} \mid \mathrm{A}}$ and rate $R=(n \hat{R}) / \ell$, as long as $R$ exceeded the capacity of $\mathcal{N}$.

Let us now sketch how our approach directly leads to a security statement for any $\mathcal{E}_{\mathrm{B} \mid \mathrm{A}}$. More specifically, we will turn things around, fix $\hat{R}$, and ask how large we have to choose $n$ such that sending $n \hat{R}$ randomly chosen classical bits through the channel $\mathcal{E}_{\mathrm{B} \mid \mathrm{A}}$ will incur an error of at least $\epsilon$. Intuitively, our goal is to effectively overflow the adversary's storage device $\mathcal{E}_{\mathrm{B} \mid \mathrm{A}}$, that is, $n$ will be chosen such that no coding scheme allows for an error less than $\epsilon$ for a fixed value of $\hat{R}$. By the results of [24] we can then bound the adversary's minentropy as $\mathrm{H}_{\min }(\mathrm{X} \mid \mathrm{BK}) \geq-\log (1-\epsilon)$. For any $\epsilon>0$, our analysis yields a bound on $\log M_{\epsilon}$ stating that if we were to transmit more than $n \hat{R}>\log M_{\epsilon}$ bits, the error is necessarily $\epsilon+\delta>\epsilon$ for some $\delta>0$, which yields the desired bound. As $\hat{R}$ is determined by an uncertainty relation, we thus know how many transmissions $n$ we have to make to obtain security. Note that allowing the adversary to perform entanglement-assisted coding only gives him additional power, and hence our analysis for entanglement-assisted coding provides a method with which explicit security parameters may be computed by means of a semi-definite program.

\section{CONCLUSION}

We have shown how a simple and powerful idea [6] for obtaining a finite blocklength converse for classical channels in terms of a hypothesis testing problem can be generalised to quantum channels and entanglementassisted codes.

This generalisation has the property that a natural restriction on codes (removing entanglement-assistance) translates into a natural restriction on the tests that can be performed in the hypothesis testing problem (they must be local). This provides a strong link between the 
extensively studied problems of channel coding and hypothesis testing (and state discrimination) of bipartite systems under locality restrictions.

Many avenues for further work are apparent to the authors: Subsection VE invites a more thorough investigation into the extent to which symmetries can be used to simplify evaluation of the bound in various special cases, and especially in the case of general memoryless channels, where one might hope for an exponential reduction in the size of the SDP, in a quantum generalisation of 13 .

While we have been able to fit the existing converse of Wang and Renner [2] precisely into our hierarchy bounds based on restricted hypothesis testing, it is not obvious to the authors what relationship exists between our converse for entanglement-assisted codes and that of Datta and Hsieh [4. We would like to know what can be said about this, particularly in light of the achievability bound given in 4 .

A limitation of our work is that we only generalise the classical bound of [6] for the case of finite input/output alphabets (as our input/output systems have finite dimension). To analyse the general case will require greater mathematical sophistication (see [7]), but would be desirable given (for example) the interest in quantum gaussian channels 31].

\section{Acknowledgments}

Matthews acknowledges the support of the Isaac Newton Trust, NSERC and QuantumWorks and would like to thank Andreas Winter, Debbie Leung, and Nilanjana Datta for useful conversations regarding this work. SW was supported by the National Research Foundation and the Ministry of Education, Singapore. We would like to thank the anonymous referees for their careful reviews of our first draft.
[1] M. Milan and N. Datta. Generalized relative entropies and the capacity of classical-quantum channels. Journal of Mathematical Physics, 50(7):072104, 2009.

[2] L. Wang and R. Renner. One-shot classical-quantum capacity and hypothesis testing. Phys. Rev. Lett., 108:200501, May 2012.

[3] J.M. Renes and R. Renner. Noisy channel coding via privacy amplification and information reconciliation. Information Theory, IEEE Transactions on, 57(11):73777385, 2011.

[4] N. Datta and M.-H. Hsieh. One-shot entanglementassisted quantum and classical communication. ArXiv e-prints, May 2011.

[5] N. Datta, M. Mosonyi, M-H. Hsieh, and F. G. S. L. Brandao. Strong converse capacities of quantum channels for classical information. To appear in IEEE Trans. Inf. Th, 2011.

[6] Y. Polyanskiy, H. V. Poor, and S. Verdú. Channel coding rate in the finite blocklength regime. IEEE Transactions on Information Theory, pages 2307-2359, 2010.

[7] Y. Polyanskiy. Saddle point in the minimax converse for channel coding. Information Theory, IEEE Transactions on, 59(5):2576-2595, 2013.

[8] E. M. Rains. Rigorous treatment of distillable entanglement. Phys. Rev. A, 60:173-178, Jul 1999.

[9] E.M. Rains. A semidefinite program for distillable entanglement. Information Theory, IEEE Transactions on, 47(7):2921 -2933, nov 2001.

[10] S. Virmani and M. B. Plenio. Construction of extremal local positive-operator-valued measures under symmetry. Phys. Rev. A, 67:062308, Jun 2003.

[11]

[12] C. H. Bennett, P. W. Shor, J. A. Smolin, and A. V. Thapliyal. Entanglement-assisted capacity of a quantum channel and the reverse Shannon theorem. Information Theory, IEEE Transactions on, 48(10):2637-2655, October 2002 .
[13] W. Matthews. A linear program for the finite block length converse of Polyanskiy, Poor, Verdú via nonsignaling codes. Information Theory, IEEE Transactions on, 58(12):7036-7044, 2012.

[14] Y. Polyanskiy and S. Verd. Arimoto channel coding converse and Rényi divergence. In Communication, Control, and Computing (Allerton), 2010 48th Annual Allerton Conference on, pages 1327-1333, Sept 2010.

[15] Naresh Sharma and Naqueeb Ahmad Warsi. Fundamental bound on the reliability of quantum information transmission. Phys. Rev. Lett., 110:080501, Feb 2013.

[16] Masahito Hayashi. Quantum Information: An Information. Springer, 2006.

[17] L. Vandenberghe and S. Boyd. Semidefinite programming. SIAM review, 38(1):49-95, 1996.

[18] A.S. Holevo. Bounds for the quantity of information transmitted by a quantum communication channel. Problemy Peredachi Informatsii, 9(3):3-11, 1973.

[19] Benjamin Schumacher and Michael D. Westmoreland. Optimal signal ensembles. Phys. Rev. A, 63:022308, Jan 2001.

[20] A.S. Holevo. The capacity of the quantum channel with general signal states. Information Theory, IEEE Transactions on, 44(1):269 -273, jan 1998.

[21] B. Schumacher and M. D. Westmoreland. Sending classical information via noisy quantum channels. Phys. Rev. A, 56:131-138, Jul 1997.

[22] C. Adami and N. J. Cerf. von neumann capacity of noisy quantum channels. Phys. Rev. A, 56:3470-3483, Nov 1997.

[23] R. König and S. Wehner. A strong converse for classical channel coding using entangled inputs. Physical Review Letters, 103:070504, 2009.

[24] R. König, S. Wehner, and J. Wullschleger. Unconditional security from noisy quantum storage. IEEE Transactions on Information Theory, 58(3):1962 - 1984, 2012.

[25] H-K. Lo and H.F. Chau. Why quantum bit commitment 
and ideal quantum coin tossing are impossible. Physica D: Nonlinear Phenomena, 120(12):177 - 187, 1998. Proceedings of the Fourth Workshop on Physics and Consumption.

[26] D. Mayers. Unconditionally secure quantum bit commitment is impossible. Phys. Rev. Lett., 78:3414-3417, Apr 1997.

[27] M. Berta, M. Christandl, F.G.S.L. Brandao, and S. Wehner. Entanglement cost of quantum channels. In Information Theory Proceedings (ISIT), 2012 IEEE International Symposium on, pages 900 -904, july 2012.

[28] M. Berta, O. Fawzi, and S. Wehner. Quantum to classical randomness extractors. In Reihaneh Safavi-Naini and Ran Canetti, editors, Advances in Cryptology CRYPTO
2012, volume 7417 of Lecture Notes in Computer Science, pages 776-793. Springer Berlin / Heidelberg, 2012.

[29] N. Ng, S. Joshi, C. Chia, C. Kurtsiefer, and S. Wehner. Experimental implementation of bit commitment in the noisy-storage model. Nature Communications, 3(1326), 2012.

[30] C. Erven, N. Ng, N. Gigov, R. Laflamme, S. Wehner, and G. Weihs. An experimental implementation of oblivious transfer in the noisy storage model. Nature Communications, 5:3418, 2014.

[31] A. S. Holevo, M. Sohma, and O. Hirota. Capacity of quantum gaussian channels. Phys. Rev. A, 59:1820-1828, Mar 1999. 